Pacific Journal of Mathematics

LES COGROUPES ET LA CONSTRUCTION DE UTUMI 


\title{
LES COGROUPES ET LA CONSTRUCTION DE UTUMI
}

\section{Labib Haddad and Yves Sureau}

\begin{abstract}
Among other results, a criterion is established, using multiplicators, for a cogroup to be obtainable by Utumi's partition method starting from a $D$-hypergroup.

Several constructions are then given of families of cogroups, finite or infinite, which are not obtainable by Utumi's method. This answers a question raised by Stephen D. Comer (J. Algebra, 89 (1984), $\mathbf{n}^{\circ} \quad 2$, 397-405).
\end{abstract}

1. Introduction. Soient $G$ un groupe, $g$ un sous-groupe quelconque de $G$ et $H=\{x g: x \in G\}$ l'ensemble des classes ("à droite") de $G$ modulo $g$. Dans le cas général, lorsque le sous-groupe $g$ n'est pas nécessairement invariant dans $G$, l'ensemble $H$ est muni naturellement d'une opération multivalente (une hyperloi de composition) qui fait de $H$ un hypergroupe. Cet hypergroupe quotient coïncide, évidemment, avec le groupe quotient $G / g$ lorsque $g$ est invariant.

Tout hypergroupe isomorphe à un hypergroupe du type précédent est appelé hypergroupe de classes à droite ou, plus simplement, $D$ hypergroupe. Cette notion a été introduite par Krasner [4] en 1937.

En 1940, Eaton [2] distingue parmi tous les hypergroupes la classe particulière des cogroupes (à droite) qu'il conçoit comme une généralisation de la notion de groupe aux structures multivalentes. Il montre que tout $D$-hypergroupe est un cogroupe et demande si la réciproque est vraie.

En 1941, puis 1944, Krasner publie deux notes [5 et 6] où, entre autres résultats, il donne une caractérisation des $D$-hypergroupes parmi les hypergroupes à l'aide de ce qu'il appelle les "permutations droites".

En 1949, Utumi [9] donne l'exemple d'un cogroupe fini ayant huit éléments et qui n'est pas un $D$-hypergroupe. Il répond ainsi, par la négative, à la question de Eaton. A cette occasion, il retrouve la notion de "permutation droite" introduite par Krasner. Il lui donne le nom de multiplicateur sous lequel on la désigne habituellement à présent. Et il retrouve, sous une forme légèrement différente, la caractérisation des $D$-hypergroupes établie par Krasner. Il fournit aussi 
un procédé général pour construire un cogroupe en partant d'un $D$ hypergroupe donné $H$ et d'une partition convenable de $H$. C'est ainsi qu'il construit son exemple à partir du groupe cyclique d'ordre huit qu'il partage convenablement en trois classes.

En 1984, en conclusion de son étude sur les polygroupes construits à l'aide de cogroupes, Comer [1] pose naturellement la question suivante: tout cogroupe provient-il d'une construction "à la Utumi"? autrement dit, tout cogroupe est-il "du type Utumi"?

Nous apportons une réponse négative à cette question.

Dans un article précédent [3], nous avions établi l'impossibilité de caractériser la classe des $D$-hypergroupes par un système d'axiomes du premier ordre, dans le langage des hypergroupes. Autrement dit, nous avions montré que le critère de Krasner-Utumi ne peut pas s'exprimer dans le langage du premier ordre.

Dans le présent travail, nous donnons un critère (nécessaire et suffisant) pour reconnaître les cogroupes "du type Utumi". Puis nous présentons deux méthodes nouvelles et générales pour construire des cogroupes. A l'aide de ces constructions et de notre critère, nous fournissons alors de multiples exemples de cogroupes (finis et infinis) qui ne sont pas "du type Utumi". En dehors de leur valeur de contreexemples, ces cogroupes enrichissent considérablement les collections connues de cogroupes et qui se limitaient à quelques rares exemplaires, en dehors de la vaste classe des $D$-hypergroupes.

En fait, pour la clarté et la simplicité, nous avons commencé par introduire et traiter des notions plus générales (hypermagmas et comagmas) où disparait l'exigence d'associativité. Bien entendu, il suffit ensuite de la réintroduire pour retrouver la situation habituelle.

Voici détaillé le contenu de l'article.

Le paragraphe 2 est destiné à rappeler ou fournir les principales définitions et fixer quelques notations.

Dans le paragraphe 3, nous introduisons les notions d'hypermagma de type $C$ et de comagma (3.1). Un comagma est, en quelque sorte, un “cogroupe non nécessairement associatif". Nous donnons un procédé général qui permet de construire tout comagma à partir d'un magma muni d'une partition convenable (3.4). Ensuite, étant donné un hypermagma $(H, *)$ quelconque et un classement $(\bar{x})_{x \in H}$ de $H$, on définit une nouvelle hyperloi sur $H$ par la formule suivante

$$
x \cdot y=x * \bar{y} .
$$


On obtient un nouvel hypermagma $(H, \cdot)$ construit à partir de $(H, *)$ à la manière de Utumi. On passe ainsi d'un original $(H, *)$ à une réplique $(H, \cdot)$ "à la Utumi" (3.5). On examine alors deux cas particuliers de la construction générale précédente. Le premier est celui où le classement donné est une partition de $H$ (3.6). Le second est celui où l'original $(H, *)$ est un $D$-hypergroupe (3.7).

Dans le premier cas, si l'original est un comagma, on donne une condition nécessaire et suffisante pour que la réplique soit aussi un comagma (3.6.4). De même, lorsque l'original est un cogroupe, on donne des conditions nécessaires et suffisantes pour que la réplique soit également un cogroupe (3.6.5).

Dans le second cas, et lorsque le classement possède en plus la propriété suivante de saturation

$e * \bar{x}=\bar{x}$ pour tout $x \in H$, où $e$ est le neutre du $D$-hypergroupe original, on dit que l'hypermagma $(H, \cdot)$, la réplique, est $d u$ type Utumi.

Dans le paragraphe 4, la notion de multiplicateur (4.1) nous sert d'outil. Nous rappelons le critère de Krasner-Utumi qui, à l'aide des multiplicateurs, caractérise les $D$-hypergroupes parmi les hypergroupoïdes (4.5). Et nous donnons nous-mêmes une nouvelle caractérisation analogue à celle de Krasner-Utumi (4.8). Nous établissons aussi un critère qui distingue parmi tous les cogroupes (ou comagmas) ceux qui sont du type Utumi (4.7).

On trouvera également dans ce paragraphe 4 des notions que nous avons jugé utile d'introduire et d'étudier: Celle de $D$-hypergroupe sous-jacent à un hypermagma quelconque (4.3). Celle de $G$-transitivité pour un hypermagma $H$ où $G$ est un groupe quelconque de multiplicateurs de $H$ (4.6). On notera, en particulier, les deux résultats suivants:

D'une part, le lemme (4.6.2) qui caractérise les groupes de multiplicateurs parmi les groupes de permutations de $H$.

D'autre part, le théorème (4.2.1.) qui donne une caractérisation remarquable des multiplicateurs spéciaux des hypermagmas $(H, *)$ de type $C$ : ce sont, précisément, les automorphismes qui préservent la partition naturelle $\{e * x: x \in H\}$.

Nous terminons le paragraphe en donnant un procédé général qui permet d'obtenir les cogroupes de type Utumi à partir des groupes munis d'une relation d'équivalence convenable (4.9 et 4.10 ).

Les deux derniers paragraphes sont essentiellement consacrés à la construction de cogroupes, finis ou infinis, qui ne sont pas du type 
Utumi et cela répond, comme on l'a déjà dit, à une question de Comer.

$\mathrm{Au}$ paragraphe 5 , on commence par mettre au point une technique générale de construction de comagmas à partir de groupes gironnés. Un groupe gironné est un groupe $G$ dans lequel on a distingué huit parties $C, C^{\prime}, F, F^{\prime}, D, D^{\prime}, E, E^{\prime}$, où les paires $\left\{D, D^{\prime}\right\}$ et $\{E$, $\left.E^{\prime}\right\}$ sont deux partitions de $G$, et les paires $\left\{C, C^{\prime}\right\}$ et $\left\{F, F^{\prime}\right\}$ sont deux partitions de $G_{*}$ (le groupe $G$ privé de son élément neutre). On prend alors une autre copie $\bar{G}$ de $G$, on choisit un élément $e$ hors de $G$ et de $\bar{G}$, on considère l'ensemble $H=\{e\} \cup G \cup \bar{G}$ que l'on munit d'une structure d'hypermagma donnée par la table (5.1). Nous donnons les conditions pour que cet hypermagma soit un cogroupe, (5.3) et (5.4). Le cas particulier des groupes taillés est d'un maniement plus simple (5.5). Nous donnons des conditions suffisantes pour que le cogroupe associé à un groupe taillé donné ne soit pas du type Utumi (5.9). Appliquée à des groupes cycliques, notre technique nous permet de construire (explicitement) trois familles de cogroupes finis et nous montrons à l'aide de notre critère qu'ils ne sont pas du type Utumi: Une première famille $H(n), n \geq 2$, où $H(n)$ posséde $8 n+1$ éléments (5.10.1). Une deuxième famille $K(n), n \geq 1$, où $K(n)$ posséde $16 n+5$ éléments (5.10.2). Une troisième famille enfin $L(n), n \geq 1$, où $L(n)$ possède $16 n+13$ éléments $(5.10 .3)$.

Dans le paragraphe 6 , le dernier, on associe à tout ensemble totalement ordonné pointé $(E, 0)$ une hyperloi comme suit:

$$
\begin{aligned}
a \cdot b & =S_{a}=\{x \in E: x<a\} & & \text { si } b<0 \\
& =\{a\} & & \text { si } b=0 \\
& =D_{a}=\{x \in E: x>a\} & & \text { si } b>0 .
\end{aligned}
$$

Puis l'on donne des conditions pour que l'hypermagma $H\langle E, 0\rangle$ ainsi défini soit un cogroupe (6.3): Lorsque $E \neq\{0\}$, l'hypermagma $H\langle E, 0\rangle$ est un cogroupe si et seulement si les deux conditions suivantes sont réunies

1. l'ensemble $E$ est dense, sans premier ni dernier élément;

2. on a $\left|S_{x}\right|=\left|S_{0}\right|$ et $\left|D_{x}\right|=\left|D_{0}\right|$ pour tout $x \in E$.

On montre ensuite (6.4) que les multiplicateurs de $H\langle E, 0\rangle$ sont précisément les automorphismes de l'ensemble ordonné $E$. D'où la condition (6.5) pour que $H\langle E, 0\rangle$ soit un cogroupe du type Utumi. Cette technique générale mise au point, nous l'appliquons à une famille d'ensembles totalement ordonnés particuliers (6.6). Pour chaque 
ordinal $\lambda>0$, on considère la somme ordinale de $\lambda$ copies de $\mathbf{R}$ :

$$
E(\lambda)=\sum_{i<\lambda} \mathbf{R}_{i}
$$

pointée au point $0=(o, o)$ origine de la première copie $\mathbf{R}_{o}$.

Nous désignons par $C(\lambda)$ l'hypermagma associé $H\langle E(\lambda), 0\rangle$. Nous montrons alors que, pour $2 \leq|\lambda| \leq 2^{\aleph_{0}}$, l'hypermagma $C(\lambda)$ est un cogroupe (infini) qui n'est pas du type Utumi.

Et, pour finir, (6.7), en prenant $\lambda=\theta$ cardinal successeur immédiat de $2^{\aleph_{0}}$, nous montrons que $C(\theta)$ est aussi un cogroupe qui n'est pas du type Utumi et qui présente une particularité nouvelle!

2. Lexique. La notion de base utilisée dans ce texte est celle d'hypermagma. Il s'agit d'un ensemble $H$ muni d'une hyperloi de composition qui fait correspondre à chaque couple $(x, y)$ d'éléments de $H$ une partie de $H$ que l'on désigne alors généralement par $x \cdot y$.

Avec cette notation, l'hyperloi se nomme encore hyperproduit.

On étend, bien sûr, à l'ensemble des parties de $H$ cet hyperproduit: pour toutes parties $A$ et $B$ de $H$, on pose

$$
A \cdot B=\{x \in H:(\exists a \in A)(\exists b \in B)(x \in a \cdot b)\} .
$$

Parmi les hypermagmas on distingue plus particulièrement:

Les hypergroupoïdes. Ce sont les hypermagmas pour lesquels on a $x \cdot y \neq \varnothing$ pour tous $x$ et $y$.

Les hypergroupes. Ce sont les hypermagmas tels que, pour tous $x$, $y, z$, on ait

$$
x \cdot(y \cdot z)=(x \cdot y) \cdot z \quad(\text { associativité })
$$

et

$$
x \cdot H=H=H \cdot x \quad \text { (reproductibilité) } .
$$

On peut noter que tout hypergroupe est un hypergroupoïde et qu'un groupe est un hypergroupe où l'hyperproduit de deux éléments quelconques se réduit à un singleton.

Soient $H$ un hypermagma et $e$ un élément de $H$.

On dit que $e$ est unité à gauche de $H$ lorsque l'on a

$$
x \in e \cdot x \text { pour tout } x \in H \text {. }
$$

On dit que $e$ est unité scalaire à droite de $H$ lorsque l'on a

$$
x=x \cdot e \text { pour tout } x \in H .
$$

On dira que $e$ est neutre (à droite) dans $H$ lorsqu'il est à la fois unité à gauche et unité scalaire à droite. 
On remarquera que si $e$ et $f$ sont neutres (à droite) dans $H$, ils sont égaux car $f \in e \cdot f=e$. Autrement dit, un hypermagma ne peut posséder qu'un seul élément neutre (à droite) au plus.

Un type d'hypergoupes joue ici un rôle privilégié, il s'agit des $h y$ pergroupes de type $C$ (à droite) introduits par Sureau [8]. Ce sont les hypergroupes possédant un élément neutre (à droite) $e$ et dans lesquels on a

$$
x \cdot y \cap x \cdot z \neq \varnothing \Rightarrow e \cdot y=e \cdot z .
$$

On se servira de la notation classique $|E|$ pour désigner le cardinal de l'ensemble $E$.

Parmi les hypergroupes de type $C$ on distingue les cogroupes (au sens de Eaton). Ce sont les hypergroupes de type $C$ vérifiant la propriété suivante:

$$
|x \cdot y|=|x \cdot z| \text { pour tous } x, y, z .
$$

Soient $H$ et $K$ des hypermagmas. On appelle morphisme de $H$ dans $K$ toute application $f: H \rightarrow K$ telle que

$$
f(x \cdot y) \subset f(x) \cdot f(y) \quad \text { pour tous éléments } x, y \text { de } H \text {. }
$$

On dit que le morphisme $f$ est bon lorsqu'on a l'égalité

$$
f(x \cdot y)=f(x) \cdot f(y) \text { pour tous éléments } x, y \text { de } H \text {. }
$$

Un isomorphisme est un morphisme bon bijectif et la bijection inverse est alors, elle aussi, un isomorphisme.

Enfin, on utilisera l'abréviation ssi pour "si et seulement si".

\section{Les comagmas.}

3.1. Quelques définitions. Soit $H$ un hypermagma. Introduisons les cinq conditions suivantes.

C1 Il existe un élément neutre (à droite) dans $H$.

C2 ("Règle de réduction à gauche"):

pour $x, y, z$, éléments de $H$, si l'on a $x y \cap x z \neq \varnothing$ alors on a $s y=s z$ pour tout $s \in H$.

C3 Pour chaque élément $x$ de $H$, la collection suivante $H(x)=$ $\{x y ; y \in H\}$ est une partition de $H$.

C4 Le cardinal de l'ensemble $x y$ ne dépend pas de $x$.

C5 ("Reproductibilité à droite"):

Pour tout élément $y$ de $H$, on a $H y=H$. 
On dira que $H$ est un hypermagma de type $C$ (à droite) lorsqu'il vérifie les trois premières conditions $\mathrm{C} 1, \mathrm{C} 2, \mathrm{C} 3$.

On dira que $H$ est un comagma (à droite) lorsqu'il vérifie les cinq conditions à la fois.

\subsection{Remarques.}

3.2.1. On notera que les notions que l'on vient d'introduire sont latéralisées "à droite".

Définir les notions analogues "à gauche" revient à étudier l'hypermagma "corrélatif" $(H, *)$ où $x * y=y x$.

Aussi nous en tiendrons-nous à l'étude de ces notions "à droite". Et, afin d'alléger, nous convenons, dès à présent, de supprimer la mention "à droite" dans toute la suite.

3.2.2. Un hypermagma de type $C$ est nécessairement un hypergroupoïde puisque $x y$ n'est jamais vide (d'après la condition $\mathrm{C} 3$ ).

3.2.3. Tout hypermagma de type $C$ vérifie automatiquement la condition de reproductibilité unilatérale suivante: $x H=H$ pour tout $x$, (d'après la même condition $\mathrm{C} 3$ ).

3.2.4. La notion d'hypergroupe de type $C$ a été introduite par Sureau [8] comme une généralisation utile de la notion de cogroupe (au sens de Eaton [2]).

Avec notre vocabulaire, un hypergroupe de type $C$ est précisément un hypergroupe qui est aussi un hypermagma de type $C$.

3.2.5. De même, un cogroupe est très exactement un comagma associatif.

3.3. Exemples.

3.3.1. Tout $D$-hypergroupe est un cogroupe [2]. Et tout cogroupe est, en particulier, un comagma.

3.3.2. Un comagma non associatif. Soit $H=\{0,1,2,3,4,5,6$, $7\}$. On définit sur $H$ une hyperloi résumée dans la table suivante, où l'on a omis la première ligne et la première colonne en bordure.

Cela se lit comme suit: par exemple

$4 \cdot 2=4 \cdot 6=\{2,6\}$ et $2 \cdot 1=2 \cdot 7=\{1,7\}$.

Et $x \cdot 0=x$ pour tout $x$.

L'élément 0 est neutre. 


\begin{tabular}{|c|c|c|c|c|}
\hline 0 & 4 & 1,7 & 3,5 & 2,6 \\
\hline 1 & 5 & 0,2 & 4,6 & 3,7 \\
2 & 6 & 1,7 & 3,5 & 0,4 \\
3 & 7 & 2,4 & 0,6 & 1,5 \\
4 & 0 & 3,5 & 1,7 & 2,6 \\
5 & 1 & 4,6 & 0,2 & 3,7 \\
6 & 2 & 1,7 & 3,5 & 0,4 \\
7 & 3 & 0,6 & 2,4 & 1,5 \\
\hline
\end{tabular}

L'hyperloi n'est pas associative puisque

$$
(4 \cdot 2) \cdot 1=\{1,7\} \quad \text { tandis que } 4 \cdot(2 \cdot 1)=\{3,5\} .
$$

\subsection{Construction des comagmas.}

3.4.1. Une construction générale. On se donne un ensemble quelconque $H$ et une partition $P$ de $H$. Pour chaque élément $x$ de $H$, on désigne par $\bar{x}$ sa classe dans la partition $P$. On se donne ensuite une loi de composition $*$ sur $H$, autrement dit, une application de $H \times H$ dans $H$ qui, à chaque couple $(x, y)$ d'éléments de $H$, fait correspondre un élément $x * y$ de $H$.

Ainsi $(H, *)$ est un magma (au sens de Bourbaki).

On dira que $(H, *)$ est un $P$-magma lorsque les trois conditions suivantes sont remplies.

A1. Il existe un élément $e$ tel que $\bar{e}=\{e\}$ et $e * x=x * e=x$ pour tout $x \in H$.

A2. Pour chaque élément $x$ de $H$, l'application $\sigma_{x}$ définie par $y \mapsto x * y$, est une permutation de $H$.

A3. On a $H * \bar{y}=H$ pour tout $y \in H$.

On définit enfin une hyperloi sur $H$ comme suit:

$$
x \cdot y=x * \bar{y}=\sigma_{x}(\bar{y}) .
$$

On obtient ainsi un hypermagma $(H, \cdot)$ dont on dira qu'il est construit sur le P-magma $(H, *)$ comme toile de fond.

3.4.2. THÉOṘ̀mE. Tout hypermagma construit sur un P-magma comme toile de fond est un comagma. 
Démonstration. Avec les notations du 3.4.1. ci-dessus, on montre que les cinq conditions du 3.1. sont satisfaites dans l'hypermagma $(H, \cdot)$.

C1. On a $\quad x \cdot e=x * \bar{e}=x * e=x$

et $e \cdot x=e * \bar{x} \supset e * x=x$.

Donc $e$ est neutre.

C2. Si $x \cdot y \cap x \cdot z \neq \varnothing$ cela veut dire que $\sigma_{x}(\bar{y})$ et $\sigma_{x}(\bar{z})$ se coupent, donc $\bar{y}$ et $\bar{z}$ se coupent, donc $\bar{y}=\bar{z}$ et, par suite, $s \cdot y=s \cdot z$ pour tout $s$.

C3. Pour chaque élément $y$, on a:

$$
e \cdot y=e * \bar{y}=\bar{y} \text {. }
$$

Donc la collection $H(e)=\{e \cdot y: y \in H\}$ n'est autre que la partition $P$ elle-même. Et, pour chaque $x$, la collection $H(x)=\{x \cdot y ; y \in H\}$ est l'image de la partition $P$ par la permutation $\sigma_{x}$. C'est donc une partition de $H$.

C4. On a $x \cdot y=\sigma_{x}(\bar{y})$ de sorte que $x \cdot y$ a le même cardinal que $\bar{y}$, indépendant de $x$.

C5. On a $H \cdot y=H * \bar{y}=H$.

3.4.3. ThÉoR Ème (réciproque). Tout comagma s'obtient par la construction générale précédente sur un P-magma comme toile de fond.

Démonstration. Soit $H$ un comagma ayant $e$ pour neutre. On désigne par $P$ la partition naturelle de $H$

$$
P=H(e)=\{e \cdot y: y \in H\} .
$$

A chaque élément $x$ de $H$ correspond sa classe $\bar{x}=e \cdot x$. Ainsi, en particulier, on a donc $\bar{e}=\{e\}$. A chaque élément $x$ de $H$ correspond aussi la partition $H(x)=\{x \cdot y: y \in H\}$ et l'on sait que $x \cdot y$ a le même cardinal que $e \cdot y$. Il existe donc au moins une permutation $\sigma_{x}$ de $H$ pour laquelle on a $\sigma_{x}(e \cdot y)=x \cdot y$ pour tout $y$. On définit une loi $*$ sur $H$ par:

$$
x * y=\sigma_{x}(y) .
$$

En choissant pour $\sigma_{e}$ la permutation identique, les trois conditions A1, A2, A 3 de la construction générale sont remplies. Autrement dit, $(H, *)$ est un $P$-magma. Et l'on a bien

$$
x \cdot y=\sigma_{x}(e \cdot y)=\sigma_{x}(\bar{y})=x * \bar{y} . \quad \text { C.Q.F.D. }
$$

3.4.4. REMARQUE. (Interprétation "géométrique" des trois conditions.) Soient $(H, *)$ un magma et $P$ une partition de $H$. 
La condition $\mathrm{A} 1$ revient à dire que le magma possède un élément neutre $e$ et que l'on a $\bar{e}=\{e\}$.

Portons notre attention sur la table de la loi du magma. On $y$ distingue, d'une part, les lignes (horizontales) et, d'autre part, les colonnes (verticales). Appelons $P$-cylindre de cette table toute réunion de colonnes ayant pour base une classe donnée quelconque de la partition $P$. La condition A2 revient ainsi à dire que chaque élément du magma figure une fois et une seule dans chacune des lignes de la table.

Et la condition $\mathrm{A} 3$ revient à dire que chaque élément du magma apparait au moins une fois dans chacun des $P$-cylindres de la table.

3.5. La construction de Utumi généralisée. On se donne un hypermagma $(H, *)$ quelconque et, à chaque élément $x$ de $H$, on associe une partie $\bar{x}$ de $H$ telle que $x \in \bar{x}$.

On dira alors que la famille $(\bar{x})_{x \in H}$ est un classement de $H$ et que $\bar{x}$ est la classe de $x$.

On définit ensuite une hyperloi $\cdot \operatorname{sur} H$ par $x \cdot y=x * \bar{y}$. On obtient ainsi un nouvel hypermagma $(H, \cdot)$. Nous dirons que l'hypermagma $(H, \cdot)$ est constuit "à la Utumi" à partir de l'hypermagma $(H, *)$ et du classement $(\bar{x})_{x \in H}$.

On notera que l'on a alors:

$$
x * y \subset x \cdot y \text { pour tous } x, y .
$$

Lorsque $(H, *)$ est un hypergroupoide et que la collection $P=\{\bar{x}: x \in$ $H$ \} est une partition de $H$, on retrouve la construction présentée par Utumi [9] qui désigne alors $(H, \cdot)$ sous le nom de "scalar partition hypergroupoïd". Nous commencerons par présenter deux cas particuliers de constructions "à la Utumi".

\subsection{Premier cas particulier.}

3.6.1. Soient $(H, *)$ un hypermagma et $P$ une partition de $H$. Pour chaque élément $x$ de $H$, on désignera par $\bar{x}$ sa classe dans la partition $P$. On dira que $(H, *)$ est un $P$-hypermagma lorsque la condition suivante est remplie:

Il existe un élément neutre e dans $(H, *)$ tel que $e * x \subset \bar{x}$ pour tout $x$.

On notera que l'on a alors $e * \bar{x}=\bar{x}$.

Tout comagma (resp. cogroupe) qui est un $P$-hypermagma se nommera P-comagma (resp. P-cogroupe). 
Soit $(H, \cdot)$ l'hypermagma construit "à la Utumi" à partir d'un $P$ hypermagma ayant $e$ pour neutre: $x \cdot y=x * \bar{y}$.

On observera, dans ce cas, que l'on a

$$
x \in e \cdot x \text { pour tout } x \text {. }
$$

Autrement dit que $e$ est unité à gauche dans $(H, \cdot)$.

On va montrer que $(H, \cdot)$ est un comagma dès lors que $(H, *)$ est un comagma et que $\bar{e}=\{e\}$.

3.6.2. Lemme. Tout hypermagma construit "à la Utumi" à partir d'un P-comagma est lui-même un comagma si $\bar{e}=\{e\}$ où e est le neutre du P-comagma.

La démonstration est analogue à celle du Théorème 3.4.2. ci-dessus.

Démonstration.

C1. On a $x \in e \cdot x$ comme on l'a déjà vu et $x \cdot e=x * \bar{e}=x * e=x$. Donc $e$ est neutre dans $(H, \cdot)$.

C2. On suppose que $x \cdot y \cap x \cdot z \neq \varnothing$. Autrement dit $x * \bar{y}$ et $x * \bar{z}$ se coupent.

Il existe donc $a \in \bar{y}$ et $b \in \bar{z}$ tels que $x * a$ et $x * b$ se coupent. Donc, $e * a=e * b$ par la condition C2 appliquée à $(H, *)$. Or $e * a \subset \bar{a}=\bar{y}$ et $e * b \subset \bar{b}=\bar{z}$. Donc $\bar{y}=\bar{z}$. Par suite

$$
s \cdot y=s * \bar{y}=s * \bar{z}=s \cdot z \quad \text { pour tout } s .
$$

C3. On veut montrer que la collection $H(x)=\{x \cdot y: y \in H\}$ est une partition de $H$.

On remarquera d'abord que si $x \cdot y$ et $x \cdot z$ se coupent ils sont égaux d'après ce qui précède.

D'autre part, pour chaque élément $t$ de $H$, il existe au moins un élément $y$ tel que $t \in x * y$. Or $x * y \subset x \cdot y$. Donc $t \in x \cdot y$. Ainsi $H(x)$ est bien une partition de $H$.

C4. On a $x \cdot y=x * \bar{y}$. Et $\bar{y}$ est réunion d'un certain nombre de classes de la partition naturelle $\{e * t: t \in H\}$ du comagma $(H, *)$. Autrement dit, il existe une partie $T$ de $H$ telle que $\bar{y}=\bigcup\{e * t: t \in$ $T\}$, où les $e * t$ sont deux à deux disjoints.

Donc $x \cdot y=x * \bar{y}=\bigcup\{x * t: t \in T\}$, et les $x * t$ sont deux à deux disjoints.

Comme, en outre, les ensembles $x * t$ et $e * t$ ont même cardinal, on voit que $x \cdot y$ a même cardinal que $\bar{y}$ indépendant de $x$.

C5. Enfin, on a $H \cdot y=H * \bar{y} \supset H * y=H$. 
Le résultat suivant est une sorte de réciproque au précédent.

3.6.3. Lemme. Soit $H$ un ensemble muni de deux hyperlois $*$ et . telles que $(H, *)$ et $(H, \cdot)$ soient tous deux des comagmas. Soit e le neutre de $(H, \cdot)$.

Les deux propriétés suivantes sont alors équivalentes.

1. Pour tous $x, y$, on $a x \cdot y=x *(e \cdot y)$.

2. Pour tous $x, y$, on $a x * y \subset x \cdot y$.

Démonstration.

$1 \Rightarrow 2$. On a $y \in e \cdot y$ donc $x * y \subset x *(e \cdot y)=x \cdot y$.

$2 \Rightarrow 1$. Soit $z \in e \cdot y$ alors $x \cdot y=x \cdot z$ (comagma). Comme $x * z \subset x \cdot z$ pour tout $z$, on a $x *(e \cdot y) \subset x \cdot y$. Réciproquement, soit $t \in x \cdot y$. Il existe alors $z$ tel que $t \in x * z$ (comagma). Donc $t \in x \cdot y \cap x \cdot z$ d'où $e \cdot y=e \cdot z$ (par "réduction"). Donc $t \in x * z \subset$ $x *(e \cdot z)=x *(e \cdot y)$. De sorte que l'on a $x \cdot y \subset x *(e \cdot y)$. D'où l'égalité $x \cdot y=x *(e \cdot y)$.

C.Q.F.D.

On peut alors regrouper ces deux derniers lemmes sous l'énoncé suivant.

3.6.4. ThÉorìme. Soient $(H, *)$ un comagma ayant e pour neutre, $(\bar{x})_{x \in H}$ un classement et $(H, \cdot)$ l'hypermagma construit "à la Utumi".

Les deux conditions suivantes sont équivalentes:

1. L'hypermagma $(H, \cdot)$ est un comagma.

2. La collection $P=\{e * \bar{y}: y \in H\}$ est une partition de $H$, le comagma $(H, *)$ est un P-comagma, $\bar{e}=\{e\}$, et $(H, \cdot)$ provient "à la Utumi" de ce P-comagma.

Démonstration. Rappelons que l'on a $x * y \subset x \cdot y$. et que $e$ est unité à gauche dans $(H, \cdot)$.

$1 \Rightarrow 2$. Soit $f$ le neutre de $(H, \cdot)$.

Alors $f \in e \cdot f=e$. Donc $f=e$ est le neutre de $(H, \cdot)$.

Appliquons le Lemme 3.6.3.

On a $x \cdot y=x *(e \cdot y)=x * \bar{y}$ pour tout $x, y$.

En particulier, on a $e \cdot y=e *(e \cdot y)=e * \bar{y}$. Donc la classe de $y$ dans la partition $P$ n'est autre que $e \cdot y$ lui-même. D'où le résultat.

$2 \Rightarrow 1$. C'est une conséquence du Lemme 3.6.2.

C.Q.F.D.

3.6.5. ThÉorème (d'associativité). Soit $(H, *)$ un cogroupe et $P$ une partition de $H$ telle que $\bar{e}=\{e\}$. On suppose que $(H, *)$ est 
un P-cogroupe et l'on désigne par $(H, \cdot)$ le comagma construit "à la Utumi" à partir de ce P-cogroupe.

Les trois propriétés suivantes sont alors équivalentes.

1. Le comagma $(H, \cdot)$ est un cogroupe.

2. Le comagma $(H, \cdot)$ est associatif.

3. Pour tous éléments $x, y$ de $H$, on $a: \overline{x * \bar{y}}=\bar{x} * \bar{y}$.

Démonstration. L'équivalence $1 \Leftrightarrow 2$ découle de la Remarque 3.2.5. $2 \Rightarrow 3$. Si $(H, \cdot)$ est associatif on a alors, en particulier,

$$
e \cdot(x \cdot y)=(e \cdot x) \cdot y
$$

donc

$$
e *(\overline{x * \bar{y}})=(e * \bar{x}) * \bar{y} .
$$

Or $e * \bar{z}=\bar{z}$ pour tout $z$ puisque $(H, *)$ est $P$-hypermagma. Donc $\overline{x * \bar{y}}=\bar{x} * \bar{y}$.

$3 \Rightarrow 2$. Réciproquement, si la condition 3 est satisfaite, on a

$$
x \cdot(y \cdot z)=x *(\overline{y * \bar{z}})=x *(\bar{y} * \bar{z}) .
$$

Or $(H, *)$ est associatif par hypothèse. Donc

$$
x \cdot(y \cdot z)=x *(\bar{y} * \bar{z})=(x * \bar{y}) * \bar{z}=(x \cdot y) \cdot z . \quad \text { C.Q.F.D. }
$$

3.6.6. L'exemple de Utumi. On désigne par $(H,+)$ le groupe cyclique d'ordre huit $H=\{0,1,2,3,4,5,6,7\}$. On considère la partition de $H$ formée des trois classes $I=\{0\}, A=\{1,4,7\}$, $B=\{2,3,5,6\}$.

Ainsi $(H,+)$ est un groupe qui est un $P$-hypermagma.

On pose $x \cdot y=x+\bar{y}$.

On obtient "à la Utumi" le comagma $(H, \cdot)$. On vérifie que la condition 3 du Théorème d'associativité 3.6.5 précédent est satisfaite. De sorte que $(H, \cdot)$ est un cogroupe.

Utumi [9] a montré que ce cogroupe n'était pas un $D$-hypergroupe.

3.6.7. Remarque. Comer et Utumi font intervenir "les inverses" d'un élément dans la condition 3 du Théorème 3.6.5 précédent. On peut s'en passer comme le montre la démonstration du théorème. On peut aussi faire le raisonnement direct suivant.

Soit $(H, *)$ un hypergroupe de type $C$ (par exemple un cogroupe) ayant $e$ pour neutre. On dit que $y$ est un inverse de $x$ lorsque $e \in$ $x * y$. Or $e \in x * y$ ssi $e \in y * x$. De sorte que $y$ est inverse de $x$ ssi $x$ est inverse de $y$. On désigne par $x^{-1}$ l'ensemble des inverses de $x$. Plus généralement on pose $X^{-1}=\bigcup_{x \in X} x^{-1}$ pour toute partie 
$X$ de $H$. On sait aussi que $e * x^{-1}=x^{-1}$ pour tout $x \in H$. Voir Eaton [2] et Sureau [8].

On se donne une partition $P$ de $H$ telle que $(H, *)$ soit un $P$ hypermagma. On suppose que l'on a $\bar{e}=\{e\}$ et $\overline{x * \bar{y}}=\bar{x} * \bar{y}$ pour tous éléments $x, y$ de $H$.

On aura automatiquement

$$
\overline{x^{-1}}=\bar{x}^{-1} \text {. }
$$

En effet, en remarquant que l'on a $e \in X$ ssi $e \in \bar{X}$, car $\bar{e}=\{e\}$, on a les équivalences suivantes:

$$
\begin{aligned}
y \in \overline{x^{-1}} & \Leftrightarrow \bar{y} \subset \overline{x^{-1}} \Leftrightarrow e \in x * \bar{y} \\
& \Leftrightarrow e \in \overline{x * \bar{y}} \Leftrightarrow e \in \bar{x} * \bar{y} \Leftrightarrow e \in \bar{y} * \bar{x} \\
& \Leftrightarrow e \in \overline{y * \bar{x}} \Leftrightarrow e \in y * \bar{x} \Leftrightarrow y \in \bar{x}^{-1} .
\end{aligned}
$$

3.7. Deuxième cas particulier. Les hypermagmas du type Utumi.

3.7.1. Soient $(H, *)$ un $D$-hypergroupe, $e$ son élément neutre et $(\bar{x})_{x \in H}$ un classement.

On suppose que l'on a

$$
e * x \subset \bar{x} \quad \text { pour tout } x .
$$

Autrement dit,

$$
e * \bar{x}=\bar{x} \quad \text { pour tout } x .
$$

On désigne par $(H, \cdot)$ l'hypermagma correspondant construit “à la Utumi"

$$
x \cdot y=x * \bar{y} .
$$

Tout hypermagma obtenu de cette manière sera dit du type Utumi.

On se souviendra qu'on a alors

$$
x * y \subset x \cdot y \text { et } x \in e \cdot x \text { pour tous } x, y .
$$

En particulier, $e$ est unité à gauche dans $(H, \cdot)$.

3.7.2. L'exemple de Utumi rappelé ci-dessus (3.6.6) est donc un cogroupe du type Utumi qui n'est pas un $D$-hypergroupe.

Comer [1] a posé la question suivante: Tout cogroupe est-il du type Utumi? Notre réponse est non! C'est ce que nous allons établir. Pour cela, nous commençons par donner une caractérisation des hypermagmas (puis des comagmas et des cogroupes) du type Utumi. 
4. Caractérisation à l'aide des multiplicateurs. Dans tout ce paragraphe, on désigne par $H$ un hypermagma.

4.1. Multiplicateurs. On appelle multiplicateur de $H$ toute permutation $\theta$ de $H$ telle que

$$
\theta(x \cdot y)=\theta(x) \cdot y \quad \text { pour tous } x \text { et } y .
$$

L'ensemble des multiplicateurs de $H$ est un sous-groupe du groupe des permutations de $H$. On l'appelle le groupe des multiplicateurs de $H$.

4.2. Multiplicateurs spéciaux. Pour tout élément $x$ de $H$, on distingue parmi les multiplicateurs ceux qui laissent $x$ invariant. On les appelle multiplicateurs spéciaux relativement à $x$. Ils forment un sous-groupe du groupe de stabilité de $x$. Lorsque $H$ est un hypermagma de type $C$ on peut donner les caractérisations suivantes des multiplicateurs spéciaux relativement à l'élément neutre.

4.2.1. Théorème. Soit $H$ un hypermagma de type $C$ ayant $e$ pour neutre. Les trois énoncés suivants sont équivalents.

1. $\sigma$ est un multiplicateur spécial relativement à $e$.

2. $\sigma$ est un automorphisme de $H$ tel que

$$
\sigma(e \cdot x)=e \cdot x \quad \text { pour tout } x \in H .
$$

3. $\sigma$ est un automorphisme de $H$ tel que

$$
\sigma(x) \in e \cdot x \text { pour tout } x \in H \text {. }
$$

Démonstration.

$1 \Rightarrow 2$. $\sigma$ étant un multiplicateur de $H$, c'est une bijection de $H$. On va montrer que c'est un morphisme bon.

Pour tout couple $(x, y)$ d'éléments de $H$, on peut écrire, d'une part

$$
\sigma(x \cdot y)=\sigma(x) \cdot y=\sigma(x) \cdot(e \cdot y),
$$

d'autre part,

$$
\sigma(e \cdot y)=\sigma(e) \cdot y=e \cdot y .
$$

Or on a $y \in e \cdot y$, donc $\sigma(y) \in \sigma(e \cdot y)=e \cdot y$ d'où $e \cdot \sigma(y)=e \cdot y$. Ainsi:

$$
\sigma(x \cdot y)=\sigma(x) \cdot(e \cdot y)=\sigma(x) \cdot(e \cdot \sigma(y))=\sigma(x) \cdot \sigma(y) .
$$

$2 \Rightarrow 3$. De $x \in e \cdot x$ on tire

$$
\sigma(x) \in \sigma(e \cdot x)=e \cdot x
$$


$3 \Rightarrow 1$. $\sigma$ étant un automorphisme de $H$, c'est une permutation de $H$. De plus on a

$$
\sigma(e)=\sigma(e \cdot e)=\sigma(e) \cdot \sigma(e)
$$

d'où l'on tire $\sigma(e)=e$; par ailleurs, pour tout couple $(x, y)$ d'éléments de $H, \sigma$ vérifie

$$
\sigma(x \cdot y)=\sigma(x) \cdot \sigma(y)=\sigma(x) \cdot(e \cdot \sigma(y)) .
$$

Or

$$
\sigma(y) \in e \cdot y \quad \text { implique } e \cdot \sigma(y)=e \cdot y \text {. }
$$

Donc

$$
\sigma(x \cdot y)=\sigma(x) \cdot(e \cdot y)=\sigma(x) \cdot y,
$$

c'est-à-dire que $\sigma$ est un multiplicateur spécial de $H$ relativement à $e$.

C.Q.F.D.

4.3. Le D-hypergroupe sous-jacent. On suppose que $H$ possède une unité à gauche $e$. On considère le groupe $M$ des multiplicateurs de $H$ et le sous-groupe $m$ des multiplicateurs spéciaux relativement à $e$.

On considère ensuite le $D$-hypergroupe quotient

$$
M / m=\{\sigma m: \sigma \in M\} .
$$

A chaque classe $\sigma m$ correspond l'élément $\sigma(e)$ de $H$. On obtient ainsi une application canonique

$$
\varphi: M / m \rightarrow H .
$$

Cette application est injective.

En effet: soient $\sigma, \tau$ deux multiplicateurs de $H$. Si $\sigma(e)=\tau(e)$ alors $\tau^{-1} \sigma(e)=e$ donc $\tau^{-1} \sigma \in m$; autrement dit $\sigma \in \tau m$, soit $\sigma m=\tau m$.

A l'aide de cette injection canonique $\varphi$ on transporte l'hyperloi du $D$-hypergroupe $M / m$ en une hyperloi sur la partie $\varphi(M / m)$ de $H$. On obtient ainsi une partie $K$ de $H$ munie d'une hyperloi $*$ de sorte que $(K, *)$ est un $D$-hypergroupe canoniquement isomorphe au $D$ hypergroupe quotient $M / \mathrm{m}$.

On appellera $(K, *)$ le $D$-hypergroupe sous-jacent à l'hypermagma $H$ relativement à $e$.

On observera ceci:

1. L'élément neutre du $D$-hypergroupe $(K, *)$ n'est autre que $e$. 
2. Soit $x \in H$. Alors $x \in K$ ssi il existe un multiplicateur $\sigma$ tel que $x=\sigma(e)$.

3. Soient $x, y$ des éléments de $K$, et $\sigma, \tau$ des multiplicateurs tels que $x=\sigma(e), y=\tau(e)$. Alors

$$
z \in x * y \quad \text { ssi } z \in \sigma m \tau(e) .
$$

4. Pour tous $x \in K$ et $y \in K$ on a

$$
x * y \subset x \cdot y .
$$

En effet: soient $z \in x * y$ et $\sigma, \tau$ des multiplicateurs tels que

$$
x=\sigma(e), \quad y=\tau(e) \quad \text { et } z=\sigma \tau(e) .
$$

Alors on a $y=\tau(e) \in e \cdot \tau(e)=e \cdot y$ et, comme $\sigma$ est un multiplicateur, on obtient $z=\sigma \tau(e)=\sigma(y) \in \sigma(e \cdot y)=\sigma(e) \cdot y=$ $x \cdot y$.

C.Q.F.D.

On peut établir plus généralement le résultat suivant.

4.4. Lemme. Soient $(H, \cdot)$ un hypermagma ayant une unité à gauche $e$, et $(K, *)$ le D-hypergroupe sous-jacent relativement $\grave{a} e$. Alors, pour tout $x \in K$ et tout $y \in H$, on a

$$
(x \cdot y) \cap K=x *[(e \cdot y) \cap K] .
$$

\section{Démonstration.}

1. Soit $z \in(x \cdot y) \cap K$ où $x \in K$. Autrement dit $z \in x \cdot y$ et $z \in K$. On choisit un multiplicateur $\sigma$ tel que $x=\sigma(e)$ et un multiplicateur $\tau$ tel que $z=\tau(e)$.

Ainsi $\tau(e)=z \in x \cdot y=\sigma(e) \cdot y=\sigma(e \cdot y)$. Soit $t=\sigma^{-1}(\tau(e))=$ $\sigma^{-1}(z)$. On a $t \in e \cdot y$ et $t=\sigma^{-1}(z)$ appartient à $K$.

De sorte que $t \in(e \cdot y) \cap K$. Or $x=\sigma(e), t=\sigma^{-1} \tau(e)$ et $z=$ $\sigma\left(\sigma^{-1} \tau(e)\right)$ donc $z \in x * t \subset x *[(e \cdot y) \cap K]$.

2. Soient $x, y$ et $z$ trois éléments de $H$ vérifiant $x \in K$ et $z \in x *[(e \cdot y) \cap K]$.

Il existe $t \in(e \cdot y) \cap K$ tel que $z \in x * t$. Donc, d'une part, on a $z \in K$.

D'autre part, soit $\tau$ un multiplicateur tel que $t=\tau(e)$. Il existe un multiplicateur $\sigma$ tel que $\sigma(e)=x$ et $z=\sigma \tau(e)$. Donc $z=\sigma(t) \epsilon$ $\sigma(e \cdot y)=\sigma(e) \cdot y=x \cdot y$. Ainsi $z \in(x \cdot y) \cap K$.

C.Q.F.D.

4.5. Le critère de Krasner-Utumi. Rappel. Nous dirons que l'hypermagma $H$ est homogène lorsque, pour tous éléments $x_{0}, x_{1}, y_{0}, y_{1}, c$ 
tels que $x_{1} \in x_{0} \cdot c$ et $y_{1} \in y_{0} \cdot c$, il existe au moins un multiplicateur $f$ de $H$ tel que

$$
f\left(x_{0}\right)=y_{0} \text { et } f\left(x_{1}\right)=y_{1} \text {. }
$$

En utilisant cette manière de parler, on a la caractérisation suivante des $D$-hypergroupes due essentiellement, et indépendamment, à Krasner [5 et 6] et Utumi [9].

Critère de Krasner-Utumi. Soit $K$ un hypergroupoïde. Afin que $K$ soit un $D$-hypergroupe il faut et il suffit qu'il soit homogène et possède une unité à gauche. (Voir aussi [3, paragraphe 9]).

4.6. Transitivité. Tout groupe de multiplicateurs de $H$ peut être considéré comme opérant sur $H$. Aussi est-il légitime de poser la définition suivante.

4.6.1. Définition. Soit $G$ un groupe quelconque de multiplicateurs de $H$. S'il existe un élément $a$ de $H$ tel que $G(a)=\{\sigma(a): \sigma \in$ $G\}=H$, on dira que $H$ est $G$-transitif.

Il faut noter que cela équivaut à la condition $G(x)=H$ pour tout $x \in H$. On peut aussi faire la remarque suivante.

4.6.2. Lemme. Soit $H$ un hypermagma et $a \in H$. Si $G$ est un groupe de permutations de $H$ tel que $G(a)=H$, les énoncés suivants sont équivalents:

1. Tout élément $\sigma$ de $G$ est un multiplicateur de $H$.

2. Pour tous éléments $\sigma$ de $G$ et $x$ de $H$, on a

$$
\sigma(a \cdot x)=\sigma(a) \cdot x \text {. }
$$

Démonstration.

$1 \Rightarrow 2$. est immédiat.

$2 \Rightarrow 1$. Soit $\sigma$ un élément de $G$.

On considère deux éléments quelconques $x$ et $y$ de $H$. Puisque $G(a)=H$, il existe $\tau$ de $G$ tel que $x=\tau(a)$.

Il s'ensuit

$$
\begin{aligned}
\sigma(x \cdot y) & =\sigma(\tau(a) \cdot y)=\sigma(\tau(a \cdot y))=\sigma \tau(a \cdot y) \\
& =(\sigma \tau(a)) \cdot y=\sigma(x) \cdot y .
\end{aligned}
$$

4.6.3. THÉORÈm (caractérisations des hypermagmas du type Utumi). Soit $H$ un hypermagma possédant une unité à gauche $e$. 
Soit $M$ le groupe de tous les multiplicateurs de $H$. Les propriétés suivantes sont équivalentes:

1. L'hypermagma $H$ est du type Utumi.

2. L'hypermagma $H$ admet un groupe $G$ de multiplicateurs pour lequel il est $G$-transitif.

3. L'hypermagma $H$ est $M$-transitif.

4. Le D-hypergroupe sous-jacent relativement à e recouvre $H$ tout entier.

Démonstration. L'équivalence $3 \Leftrightarrow 4$ découle des définitions ( 4.3 et 4.6.1.).

$2 \Rightarrow 3$. Si $H$ est $G$-transitif il est a fortiori $M$-transitif.

$3 \Rightarrow 2$. Evidemment!

$1 \Rightarrow 3$. Par hypothèse, il existe une hyperloi $*$ sur $H$, un classement $(\bar{x})_{x \in H}$ de $H$ tels que $(H, *)$ soit un $D$-hypergroupe et $x \cdot y=$ $x * \bar{y}$ pour tous $x$ et $y$.

Mais tout multiplicateur $\theta$ du $D$-hypergroupe $(H, *)$ est multiplicateur de l'hypermagma $(H, \cdot)$. En effet:

$$
\theta(x \cdot y)=\theta(x * \bar{y})=\theta(x) * \bar{y}=\theta(x) \cdot y .
$$

Or d'après le critère de Krasner-Utumi, pour chaque $x \in H$, il existe un multiplicateur du $D$-hypergroupe $(H, *)$ tel que $\theta(e)=x$. Donc $(H, \cdot)$ est $M$-transitif.

$4 \Rightarrow 1$. Soit $(K, *)$ le $D$-hypergroupe sous-jacent à $(H, \cdot)$ relativement à $e$.

Par hypothèse, on a $K=H$.

Ainsi, d'après le Lemme 4.4, on a:

$$
x \cdot y=x *(e \cdot y) .
$$

En particulier, pour $x=e$, on obtient

$$
e \cdot y=e *(e \cdot y)
$$

On pose $\bar{x}=e \cdot x$ pour tout $x \in H$. Ainsi $(\bar{x})_{x \in H}$ est un classement de $(H, \cdot)$ et on a:

$$
e * \bar{x}=\bar{x} \quad \text { et } \quad x \cdot y=x * \bar{y}
$$

pour tous $x$ et $y$ de $H$.

Donc l'hypermagma $(H, \cdot)$ est bien du type Utumi. C.Q.F.D.

4.7. Corollaire. Un comagma (resp. cogroupe) ayant e pour neutre est du type Utumi si et seulement si, pour tout élément $x$, il existe au moins un multiplicateur $\theta$ pour lequel $\theta(e)=x$. 
Voici maintenant un critère analogue à celui de Krasner-Utumi pour les $D$-hypergroupes.

4.8. Corollaire. Soit $H$ un hypermagma possédant une unité à gauche $e$. Les deux énoncés suivants sont équivalents

1. L'hypermagma $H$ est un D-hypergroupe.

2. Il existe un groupe $G$ de multiplicateurs de $H$ tel que

(i) $H$ soit $G$-transitif

(ii) $g(x)=e \cdot x$ pour tout $x \in H$ où $g=\{\sigma \in G: \sigma(e)=e\}$.

De plus, dans ce cas, $H$ est isomorphe au D-hypergroupe quotient $G / g$.

$1 \Rightarrow 2$. Par hypothèse il existe un groupe $G_{1}$ admettant un sousgroupe $g_{1}$ tel que, à un isomorphisme près, on ait $H=G_{1} / g_{1}$.

A tout $\alpha \in G_{1}$ correspond un multiplicateur $\theta_{\alpha}$ de $H$ défini par

$$
\theta_{\alpha}\left(x_{1} g_{1}\right)=\alpha x_{1} g_{1} \text { pour tout } x_{1} \in G_{1} \text {, }
$$

et l'application

$$
\begin{aligned}
\theta: G_{1} & \rightarrow S(H) \\
\alpha & \rightsquigarrow \theta_{\alpha}
\end{aligned}
$$

de $G_{1}$ dans le groupe symétrique $S(H)$ de $H$ est un morphisme de groupes dont l'image $G$ est un groupe de multiplicateurs de $H$.

La reproductibilité de $G_{1}$ assure immédiatement que $H$ est $G$ transitif. L'ensemble $g$ des multiplicateurs spéciaux de $G$ relativement à $e$ correspond aux éléments $\alpha$ de $G_{1}$ vérifiant $\alpha g_{1}=g_{1}$.

Autrement dit

$$
g=\left\{\theta_{\alpha}: \alpha \in g_{1}\right\}=\left\{\theta_{\alpha} \in G: \theta_{\alpha}(e)=e\right\} .
$$

L'ensemble $g$ est un sous-groupe de $G$.

Soit alors $x=x_{1} g_{1}$ et $e=g_{1}$. On a $g(x)=g_{1} x_{1} g_{1}=e \cdot x$.

$2 \Rightarrow 1$. En reprenant les notations du paragraphe 4.3 , le Théorème 4.6.3. précédent établit que $\varphi$ est une bijection. De plus, pour tout couple $(\sigma, \tau)$ d'éléments de $G$, on a

$$
\varphi(\sigma g \tau g)=\sigma g \tau g(e)=\sigma(g(\tau(e)))
$$

et comme, par hypothèse, $g(\tau(e))=e \cdot \tau(e)$, on a

$$
\varphi(\sigma g \tau g)=\sigma(e \cdot \tau(e))=\sigma(e) \tau(e)=\varphi(\sigma g) \varphi(\tau g) .
$$

Ainsi $\varphi$ définit un isomorphisme entre le $D$-hypergroupe $G / g$ et l'hypermagma $(H, \cdot)$.

C.Q.F.D. 
Dans la construction qui suit, on aura besoin de la notion de soushypergroupe inversible à droite. On pourra consulter Sureau [7] pour cela.

4.9. Une construction. Soit $G$ un groupe multiplicatif ayant $e$ pour élément neutre. On considère une relation d'équivalence $R$ sur $G$ vérifiant la propriété suivante

$$
R(x R(y))=R(x) R(y) \text { pour tous } x, y \text { de } G .
$$

On pose:

$$
g=R(e), \quad x * y=x R(y) .
$$

On a alors ceci

1. $g$ est un sous-groupe de $G$.

2. $(G, *)$ est un hypergroupe.

3. $(g, *)$ est un sous-hypergroupe inversible à droite de $(G, *)$. On considère le $D$-hypergroupe $G / g$ et le classement défini par $\overline{x g}=$ $R(x g)$.

4. Ce classement est une partition de $G / g$ qui vérifie la propriété

$$
\overline{x g \overline{y g}}=\overline{x g} \overline{y g} \text {. }
$$

On pose enfin

$$
x g \cdot y g=x g R(y g) \text {. }
$$

5. Le cogroupe de type Utumi $(G / g, \cdot)$ est isomorphe à

$$
(G, *) /(g, *) \text {. }
$$

Démonstrations.

1. Soient $a \in g, b \in g, x=a^{-1} b$.

Alors

$$
\begin{aligned}
e \in g & =R(b)=R(a x) \subset R(a R(x))=R(a) R(x) \\
& =R(e) R(x)=R(e R(x))=R(R(x))=R(x) .
\end{aligned}
$$

Ainsi $e \in R(x)$ donc $x \in g$. Autrement dit

$$
g^{-1} g \subset g \text {. }
$$

Donc $g$ est un sous-groupe de $G$.

2. Se démontre sans détour.

3. Il faut montrer que $x \in y * g$ implique $y \in x * g$. Or on a $y * g=y g$, de sorte que:

$$
x \in y * g \Leftrightarrow y^{-1} x \in g \Leftrightarrow x^{-1} y \in g \Leftrightarrow y \in x * g .
$$


4. On remarque tout d'abord que, pour tout $x \in G$, on a

$$
R(x g)=R(x R(e))=R(x) R(e)=R(x) g .
$$

Donc, d'une part, on a

$$
R(x g) g=R(x) g g=R(x) g,
$$

et d'autre part, pour tout $y \in G$, on a

$$
\begin{aligned}
& R(x g) \text { coupe } R(y g) \Leftrightarrow R(x) g \text { coupe } R(y) g \\
& \Leftrightarrow\left(\exists x^{\prime} \in R(x)\right)\left(\exists y^{\prime} \in R(y)\right)\left(x^{\prime} g=y^{\prime} g\right) \\
& \Rightarrow R(x g)=R(y g) \Rightarrow R(x g) \text { coupe } R(y g) .
\end{aligned}
$$

De sorte que le classement est bien une partition de $G / g$. Enfin

$$
R(x g R(y g))=R(x g) R(y g) \text {. }
$$

5. Le Théorème 3.6 .5 montre que $(G / g, \cdot)$ est un cogroupe. Enfin, l'application $x * g \mapsto x g$ est un isomorphisme de l'hypergroupe $(G, *) /(g, *) \operatorname{sur}(G / g, \cdot)$.

C.Q.F.D.

Voici, à présent, un résultat qui montre que tout cogroupe du type Utumi s'obtient par une construction du type précédent.

4.10. ThÉORème. Soit $G$ un groupe multiplicatif ayant e pour élément neutre et $g$ un sous-groupe de $G$. On considère un classement du D-hypergroupe $G / g$ où $\theta(x g)$ désigne la classe de $x g$. On pose

$$
x g \cdot y g=x g \theta(y g)
$$

et on suppose que $(G / g, \cdot)$ est un cogroupe.

On pose enfin

$$
R(x)=g \theta(x g)
$$

Alors on a ceci.

1. $R$ est une relation d'équivalence sur $G$ qui vérifie la propriété

$$
R(x R(y))=R(x) R(y) .
$$

2. $R(e)=g$.

3. La construction précédente (4.9) redonne le cogroupe $(G / g, \cdot)$ isomorphe à $(G, *) /(g, *)$.

Démonstration. Notons $*$ l'hyperloi du $D$-hypergroupe $G / g$.

D'après le Théorème 3.6.4, on peut dire ceci:

La collection $P=\{g * \theta(x g): x \in G\}$ est une partition de $G / g$; le $D$-hypergroupe $G / g$ est un $P$-cogroupe; on a $\theta(g)=\{g\}$ et $(G / g, \cdot)$ 
provient "à la Utumi" de ce $P$-cogroupe. De sorte que le cogroupe $(G / g, \cdot)$ est du type Utumi.

Le théorème d'associativité 3.6 .5 montre que l'on a alors $\theta(x g * \theta(y g))=\theta(x g) * \theta(y g)$ pour tous $x$ et $y$ de $G$.

1. L'ensemble $\{g \theta(x g): x \in G\}$ est une partition de $G$ donc $R$ est bien une équivalence sur $G$.

De plus,

$$
\begin{aligned}
R(x R(y)) & =R(x g \theta(y g))=g \theta(x g \theta(y g)) \\
& =g \theta(x g) \theta(y g)=g \theta(x g) g \theta(y g)=R(x) R(y) .
\end{aligned}
$$

D'où le résultat

2. et 3. sont sans détour.

C.Q.F.D.

5. Comagma associé à un groupe gironné.

5.1. Une construction. On se donne un groupe $G$ ayant 0 pour élément neutre et noté additivement.

On pose $G_{*}=G \backslash\{0\}$ et on se donne deux partitions de l'ensemble $G_{*}$ que l'on désignera comme suit:

$$
\left\{C, C^{\prime}\right\} \text { d'une part }
$$

et

$$
\left\{F, F^{\prime}\right\} \quad \text { d'autre part. }
$$

Cela suppose que $G$ possède au moins trois éléments.

On se donne aussi deux partitions de $G$ lui-même que l'on désignera comme suit:

$$
\left\{D, D^{\prime}\right\} \quad \text { d'une part }
$$

et

$$
\left\{E, E^{\prime}\right\} \quad \text { d'autre part. }
$$

On dira que $\left(G ; C, C^{\prime}, F, F^{\prime}, D, D^{\prime}, E, E^{\prime}\right)$ est un groupe gironné. On prend une autre copie $\bar{G}$ de $G$, disjointe de $G$. On choisit enfin un élément $e$ hors de $G$ et de $\bar{G}$.

Si $x \in G$, on désignera par $\bar{x}$ l'élément correspondant de $\bar{G}$. De même, si $X \subset G$, on désignera par $\bar{X}$ la partie correspondante de $\bar{G}$. On pose $H=\{e\} \cup G \cup \bar{G}$.

On définit enfin une hyperloi sur $H$ résumée dans la table suivante.

\begin{tabular}{|l|lll|lcc|}
\hline$e$ & & & $G$ & & $\bar{G}$ \\
\hline$x$ & $e$ & $x+C$ & $\overline{x+D}$ & & $x+C^{\prime}$ & $\overline{x+D^{\prime}}$ \\
\hline $\bar{x}$ & & $x+E^{\prime}$ & $\overline{x+F^{\prime}}$ & $e$ & $x+E$ & $\overline{x+F}$ \\
\hline
\end{tabular}


Sur une colonne, on lit de haut en bas les produits $e \alpha, x \alpha, \bar{x} \alpha$ (pour tout $x \in G)$, où $\alpha=e$ pour la colonne de gauche, $\alpha$ est élément de $G$ pout la colonne centrale et $\alpha$ est élément de $\bar{G}$ pour la colonne de droite.

Voici quelques explications. La collection $H(e)=\{\{e\}, G, \bar{G}\}$ est une partition de $H$. Pour chaque élément $a$ de $H$, l'hyperproduit $a y$ ne dépend que de la classe à laquelle appartient $y$ dans cette partition.

En particulier, on a

$$
a e=a \quad \text { et } \quad a \in e a .
$$

On dira que l'hypermagma $H$ ainsi construit est associé au groupe gironné.

5.2. LeMME. L'hypermagma $H$ associé à un groupe gironné est de type $\mathrm{C}$ et vérifie la reproductibilité (à gauche et à droite).

\section{Démonstration.}

C1. L'élément $e$ est neutre dans $H$.

C2. Si $a y \cap a z \neq \varnothing$ alors $y$ et $z$ appartiennent à la même classe de la partition $H(e)$. Autrement dit, ou bien $y=z=e$, ou bien $y$ et $z$ appartiennent tous les deux à $G$, ou tous les deux à $\bar{G}$. On a donc $s y=s z$ pour chaque élément $s$ de $H$.

C3. La collection $H(a)=\{a y: y \in H\}=\{\{a\}, a G, a \bar{G}\}$ est une partition de $H$ pour chaque élément $a$ de $H$.

Enfin, on a bien $a H=H=H a$ pour tout élément $a$ de $H$.

5.3. LeMme. L'hypermagma $H$ associé au groupe gironné $(G ; C$, $\left.C^{\prime}, F, F^{\prime}, D, D^{\prime}, E, E^{\prime}\right)$ est un comagma si et seulement si on a les égalités suivantes entre cardinaux

$$
1+|C|+|D|=\left|E^{\prime}\right|+\left|F^{\prime}\right|=|G|
$$

et

$$
1+|E|+|F|=\left|C^{\prime}\right|+\left|D^{\prime}\right|=|G|
$$

En effet, ces égalités sont nécessaires et suffisantes pour que la condition C4 (paragraphe 3.1.) soit satisfaite.

5.4. Associativité. Voici des conditions nécessaires et suffisantes pour que l'hypermagma $H$ associé au groupe gironné soit associatif. 
Ces conditions sont formées des six paires suivantes:
(1) $(C+D) \cup\left(D+F^{\prime}\right)=G$, $(F+E) \cup\left(E+C^{\prime}\right)=G$,
(2) $\left(C+C^{\prime}\right) \cup(D+E)=G_{*}$, $\left(F+F^{\prime}\right) \cup(E+D)=G_{*}$,
(3) $\left(C^{\prime}+C\right) \cup\left(D^{\prime}+E^{\prime}\right)=G_{*}$, $\left(F^{\prime}+F\right) \cup\left(E^{\prime}+D^{\prime}\right)=G_{*}$,
(4) $\left(C^{\prime}+D\right) \cup\left(D^{\prime}+F^{\prime}\right)=G$, $\left(F^{\prime}+E\right) \cup\left(E^{\prime}+C^{\prime}\right)=G$,
(5) $\left(C^{\prime}+C^{\prime}\right) \cup\left(D^{\prime}+E\right)=G$, $\left(F^{\prime}+F^{\prime}\right) \cup\left(E^{\prime}+D\right)=G$,
(6) $\left(C^{\prime}+D^{\prime}\right) \cup\left(D^{\prime}+F\right)=G$, $\left(F^{\prime}+E^{\prime}\right) \cup\left(E^{\prime}+C\right)=G$.

On peut les ramasser sous la forme matricielle commode suivante
(1) et (2)
$\left(\begin{array}{ll}C & D \\ E & F\end{array}\right)$
$\left(\begin{array}{cc}C^{\prime} & D \\ E & F^{\prime}\end{array}\right)=\left(\begin{array}{cc}G_{*} & G \\ G & G_{*}\end{array}\right)$
(3) et (6)
$\left(\begin{array}{ll}C^{\prime} & D^{\prime} \\ E^{\prime} & F^{\prime}\end{array}\right)$
$\left(\begin{array}{cc}C & D^{\prime} \\ E^{\prime} & F\end{array}\right)=\left(\begin{array}{cc}G_{*} & G \\ G & G_{*}\end{array}\right)$
(4) et (5)
$\left(\begin{array}{ll}C^{\prime} & D^{\prime} \\ E^{\prime} & F^{\prime}\end{array}\right)$
$\left(\begin{array}{l}C^{\prime} \\ E\end{array}\right.$
$\left.\begin{array}{l}D \\ F^{\prime}\end{array}\right)=\left(\begin{array}{l}G \\ G\end{array}\right.$
$\left.\begin{array}{l}G \\ G\end{array}\right)$.

5.4.1. LeMME. L'hypermagma $H$ associé au groupe gironné $(G ; C$, $\left.C^{\prime}, F, F^{\prime}, D, D^{\prime}, E, E^{\prime}\right)$ est associatif si et seulement si les six paires de conditions précédentes sont remplies.

La démonstration, technique mais sans détour, est renvoyée en appendice.

5.4.2. Remarque. Lorsque les six paires de conditions d'associativité sont satisfaites, on a nécessairement

$$
C=-C, \quad F=-F, \quad E^{\prime}=-D, \quad D^{\prime}=-E
$$

(où $-X$ désigne l'ensemble des opposés des éléments de $X$ dans le groupe $G$ ).

En effet: La condition (2) implique que $0 \notin C+C^{\prime}$, donc $-C \subset C$ et $-C^{\prime} \subset C^{\prime}$ d'où l'égalité $C=-C$.

De même $F=-F$.

D'autre part, toujours d'après (2), on doit avoir $0 \notin D+E$, donc $-D \subset E^{\prime}$. De même la condition (3) implique que l'on a $0 \notin D^{\prime}+E^{\prime}$, donc $-D^{\prime} \subset E$. Or $\left\{E, E^{\prime}\right\}$ et $\left\{-D,-D^{\prime}\right\}$ sont des partitions de $G$. D'où $E^{\prime}=-D$ et $E=-D^{\prime}$ (i.e.) $D^{\prime}=-E$.

C.Q.F.D.

5.5. Un cas particulier. Groupe taillé. On considère une partition $\{S, T\}$ de $G_{*}$ vérifiant les deux conditions suivantes

$$
(S+T) \cup T=G_{*} \quad \text { et } \quad T+T=G .
$$


On dira que $(G ; S, T)$ est un groupe taillé.

On prend alors $U=S \cup\{0\}$ et

$$
\begin{array}{ll}
C=S, & C^{\prime}=T, \\
F=S, & F^{\prime}=T, \\
D=U, & D^{\prime}=T, \\
E=T, & E^{\prime}=U .
\end{array}
$$

On obtient alors un groupe gironné particulier

$$
(G ; S, T, S, T, U, T, T, U) \text {. }
$$

On dira que l'hypermagma associé à ce groupe gironné est l'hypermagma associé au groupe taillé $(G ; S, T)$.

On va vérifier que les six paires de conditions d'associativité sont toujours satisfaites dans ce cas.

En effet: On commence par remarquer que l'on a $S+T \subset G_{*}$ par l'hypothèse. Donc $S=-S$ et $T=-T$. Ainsi $0 \in S+S$ et $(T+S) \cup T=G_{*}$.

Et donc $U+T=T+U=G_{*}$. On vérifie ensuite, une à une, les six paires de conditions:

$$
\begin{aligned}
& (C+D) \cup\left(D+F^{\prime}\right)=(S+U) \cup(U+T)=\{0\} \cup G_{*}=G, \\
& (F+E) \cup\left(E+C^{\prime}\right)=(S+T) \cup(T+T)=G, \\
& \left(C+C^{\prime}\right) \cup(D+E)=(S+T) \cup(U+T)=G_{*}, \\
& \left(F+F^{\prime}\right) \cup(E+D)=(S+T) \cup(T+U)=G_{*}, \\
& \left(C^{\prime}+C\right) \cup\left(D^{\prime}+E^{\prime}\right)=(T+S) \cup(T+U)=G_{*}, \\
& \left(F^{\prime}+F\right) \cup\left(E^{\prime}+D^{\prime}\right)=(T+S) \cup(U+T)=G_{*}, \\
& \left(C^{\prime}+D\right) \cup\left(D^{\prime}+F^{\prime}\right)=(T+U) \cup(T+T)=G, \\
& \left(F^{\prime}+E\right) \cup\left(E^{\prime}+C^{\prime}\right)=(T+T) \cup(U+T)=G, \\
& \left(C^{\prime}+C^{\prime}\right) \cup\left(D^{\prime}+E\right)=(T+T) \cup(T+T)=G, \\
& \left(F^{\prime}+F^{\prime}\right) \cup\left(E^{\prime}+D\right)=(T+T) \cup(U+U)=G, \\
& \left(C^{\prime}+D^{\prime}\right) \cup\left(D^{\prime}+F\right)=(T+T) \cup(T+S)=G, \\
& \left(F^{\prime}+E^{\prime}\right) \cup\left(E^{\prime}+C\right)=(T+U) \cup(U+S)=G *\{0\}=G .
\end{aligned}
$$

On a donc le résultat suivant:

5.5.1. LEMME. L'hypermagma associé à un groupe taillé est toujours un hypergroupe de type $C$.

Voici, à présent, une condition pour que l'hypergroupe associé à un groupe taillé soit un cogroupe. 
5.6. THÉORÈme. L'hypergroupe $H$ associé au groupe taillé $(G ; S$, T) est un cogroupe si et seulement si l'on a

$$
2|S|+2=2|T|=|G| \text {. }
$$

Démonstration. On utilise le Lemme 5.3.

La condition est nécessaire car, avec les notations de ce lemme, on doit avoir

$$
1+|C|+|D|=\left|C^{\prime}\right|+\left|D^{\prime}\right|=|G|
$$

autrement dit

$$
1+|S|+|S|+1=|T|+|T|=|G| .
$$

La condition est suffisante car on aura toujours

$$
1+|E|+|F|=1+|T|+|S|=|G|
$$

et

$$
\left|E^{\prime}\right|+\left|F^{\prime}\right|=1+|S|+|T|=|G| .
$$

ReMARQue. Lorsque le groupe $G$ est fini, $H$ est un cogroupe

$$
\text { ssi } 2|S|+2=|G| \text { ssi } 2|T|=|G| \text {. }
$$

Lorsque le groupe $G$ est infini, $H$ est un cogroupe

$$
\text { ssi }|S|=|T|=|G| \text {. }
$$

Les deux lemmes qui suivent nous serviront à établir des critères suffisants pour qu'un cogroupe associé à un groupe taillé ne soit pas du type Utumi.

5.7. Lemme. Soient $(G ; S, T)$ un groupe taillé, $H$ l'hypergroupe associé, 0 l'élément neutre de $G$, e l'élément neutre de $H$.

On suppose la condition suivante satisfaite.

$$
|S \cap(x+S)|<|S| \text { pour tout } x \in G_{*} .
$$

Il n'existe alors aucun multiplicateur $\theta$ tel que $\theta(e)=0$.

Démonstration. Raisonnons par l'absurde.

Soit $\theta$ un multiplicateur tel que $\theta(e)=0$.

Alors

$$
\theta(G)=\theta(e G)=\theta(e) G=0 G=\{e\} \cup S \cup \bar{U} \quad \text { où } U=S \cup\{0\} .
$$

Soit $Y=\theta^{-1}(S)$. Ainsi $Y \subset G$. 
On considère alors les deux intersections suivantes:

$$
\begin{aligned}
& I=(e G) \cap \bigcap\{y G: y \in Y\}, \\
& J=(0 G) \cap \bigcap\{x G: x \in S\} .
\end{aligned}
$$

On a donc $\theta(I)=J$. Les deux ensembles $I$ et $J$ devraient donc avoir même cardinal.

Or, on va voir que $|I| \leq 1$ et $|J| \geq 2$.

En effet: Supposons que $I$ renferme les deux éléments $a$ et $b$. Alors $a \in G$ et $a \in y+S$ pour tout $y \in Y$, donc $y \in(a-S)=a+S$. Autrement dit $Y \subset a+S$. De même, on aura $Y \subset b+S$. Donc

$$
|(a+S) \cap(b+S)| \geq|Y|=|S| .
$$

En posant $d=-b+a$, on aura donc

$$
\begin{aligned}
|S| & \leq|(a+S) \cap(b+S)| \\
& \leq|(-b+a+S) \cap(-b+b+S)|=|(d+S) \cap S| .
\end{aligned}
$$

D'après la condition (5.7.1), on a donc $d=0$ soit $a=b$.

Ainsi $|I| \leq 1$.

D'autre part, on a $e \in J$ car $e \in x G$ pour tout $x \in G$. Et on a aussi $\overline{0} \in J$ : en effet, $0 \in U=D$ donc $\overline{0} \in \bar{D}$ donc $\overline{0} \in 0 G$; et, pour chaque $x \in S=C$, on a $(-x) \in S \subset D$ donc $0 \in(x+D)$ d'où $\overline{0} \in \overline{x+D} \subset x G$.

Ainsi $|J| \geq 2$.

5.8. Lemme. Soient $(G ; S, T)$ un groupe taillé, $H$ l'hypergroupe associé, 0 l'élément neutre de $G$, e l'élément neutre de $H$.

On pose

$$
\begin{aligned}
& A=\bigcap\{x+S: x \in S\}, \\
& B=\bigcap\{x+T: x \in S \cup\{0\}\} .
\end{aligned}
$$

On suppose que l'on a l'inégalité suivante:

$$
|A|>1+|B| \text {. }
$$

Il n'existe alors aucun multiplicateur $\theta$ de $H$ tel que $\theta(e)=\overline{0}$.

Démonstration. On raisonne par l'absurde.

Soit $\theta$ un multiplicateur de $H$ tel que $\theta(e)=\overline{0}$.

Alors:

$$
\theta(\bar{G})=\theta(e \bar{G})=\theta(e) \bar{G}=\overline{0} \bar{G}=\{e\} \cup T \cup \bar{S}
$$


Ainsi $\theta^{-1}(\bar{S}) \subset \bar{G}$. Soit $\bar{Y}=\theta^{-1}(\bar{S})($ avec $Y \subset G)$.

On considère les deux intersections suivantes

$$
\begin{aligned}
& I=e \bar{G} \cap \bigcap\{\bar{y} \bar{G}: y \in Y\}, \\
& J=\overline{0} \bar{G} \cap \bigcap\{\bar{x} \bar{G}: x \in S\} .
\end{aligned}
$$

Ainsi $\theta(I)=J$. On devrait donc avoir $|I|=|J|$. On va montrer cependant qu'il n'en est rien.

En effet: On a $e \bar{G}=\bar{G}$ et $\bar{y} \bar{G}=\{e\} \cup(y+T) \cup(\overline{y+S})$ pour tout $y \in Y$. De sorte que l'on a:

$$
I=\bigcap\{\overline{y+S}: y \in Y\} .
$$

D'autre part, on a:

$$
\overline{0} \bar{G}=\{e\} \cup T \cup \bar{S} \text { et } \bar{x} \bar{G}=\{e\} \cup(x+T) \cup(\overline{x+S})
$$

pour tout $x \in S$.

Donc

$$
J=\{e\} \cup B \cup \bar{N} \quad \text { où } N=\bigcap\{(x+S): x \in S \cup\{0\}\} .
$$

Vérifons que $N$ est vide, par l'absurde.

Si on avait $a \in N$, on aurait $a \in(x+S)$ pour tout $x \in S \cup\{0\}$. En particulier, on aurait donc $a \in S$ donc aussi $a \in(a+S)$, autrement dit $0 \in S \subset G_{*}$ ce qui est absurde.

On a donc, finalement:

$$
J=\{e\} \cup B
$$

On distingue alors deux cas:

1 er cas. Si $I$ est vide, alors $|I|=0$ et $|J| \geq 1$.

2 ème cas. Si $I$ n'est pas vide.

Il existe alors un élément $a \in \bigcap\{y+S: y \in Y\}$. Donc $Y \subset a-S=$ $a+S(\operatorname{car} S=-S)$. Ainsi

$$
\begin{aligned}
& \bigcap\{y+S: y \in Y\} \supset \bigcap\{y+S: y \in a+S\} \\
& \quad=\bigcap\{a+x+S: x \in S\}=a+\bigcap\{x+S: x \in S\} \\
& =a+A .
\end{aligned}
$$

Or, par hypothèse on a $|A|>1+|B|$. D'où $|I| \geq|A|>1+|B|=|J|$ (car $e \notin B$ ). Dans les deux cas, on a donc bien $|I| \neq|J|$. C.Q.F.D. 
5.9. ThÉorème. Soit $(G ; S, T)$ un groupe taillé tel que la propriété suivante soit satisfaite.

$$
2|S|+2=2|T|=|G|,
$$

et soit $H$ le cogroupe associé. Lorsque l'une ou l'autre des conditions 5.7.1 ou 5.8.1 est satisfaite, le cogroupe $H$ n'est pas du type Utumi.

Cela découle de notre critère 4.7 à travers les deux Lemmes 5.7 et 5.8 ci-dessus.

5.10. Exemples de cogroupes finis qui ne sont pas du type Utumi.

5.10.1. Une première famille. On se fixe un entier $n \geq 2$, on pose $m=4 n$ et on considère le groupe cyclique $G=\mathbf{Z} / m \mathbf{Z}$.

On pose

$$
S=\{n+1, \ldots, 3 n-1\} \quad \text { et } \quad T=\{1, \ldots, n ; 3 n, \ldots, 4 n-1\} \text {. }
$$

On obtient un groupe taillé $(G ; S, T)$ qui vérifie les conditions 5.6.1 et 5.7.1.

En effet:

$1^{\circ}$ On a $|S|=2 n-1,|T|=2 n,|G|=4 n$, et la condition 5.6.1 est vérifiée.

$2^{\circ}$ On calcule $T+T$

$T+T=\{2, \ldots, 2 n ; 3 n+1, \ldots, 5 n-1 ; 6 n, \ldots, 8 n-2\}$. Soit, en calculant modulo $4 n, T+T=\{2, \ldots, 2 n ; 3 n+1, \ldots, 4 n ; 1, \ldots$, $n-1 ; 2 n, \ldots, 4 n-2\}=G$.

$3^{\circ}$ On calcule $S+T$

$$
\begin{aligned}
S+T & =\{n+2, \ldots, 4 n-1 ; 4 n+1, \ldots, 7 n-2\} \\
& =\{n+2, \ldots, 4 n-1 ; 1, \ldots, 3 n-2\}
\end{aligned}
$$

et, comme $n \geq 2$, on a $3 n-2 \geq n+2$, donc $S+T=\{1, \ldots, 4 n-1\}=$ $G_{*}$. Ainsi $(G ; S, T)$ est bien un groupe taillé.

$4^{\circ}$ Enfin, pour $x \neq 0$, on a $x+S \neq S$ car $S$ est un "arc" dans le groupe cyclique! Donc $|S \cap(x+S)|<|S|$.

Et la condition 5.7.1 est vérifiée.

On désigne par $H(n)$ le cogroupe associé.

La famille $H(n), n \geq 2$, est donc une famille de cogroupes finis qui ne sont pas du type Utumi. 
Le cogroupe $H(n)$ possède $2 m+1=8 n+1$ éléments. Le plus petit de la famille, $H(2)$, possède 17 éléments. Voir sa table ci-dessous en appendice.

5.10.2 Une deuxième famille. On se fixe un entier $n \geq 1$, on pose $m=8 n+2$ et on considère le groupe cyclique $G=\mathbf{Z} / m \mathbf{Z}$.

On pose

$$
\begin{aligned}
& S=\{n+1, \ldots, 3 n ; 5 n+2 \ldots, 7 n+1\}, \\
& T=\{1, \ldots, n ; 3 n+1, \ldots, 5 n+1 ; 7 n+2, \ldots, 8 n+1\} .
\end{aligned}
$$

On obtient un groupe taillé $(G ; S, T)$ qui vérifie les conditions 5.6.1 et 5.8.1.

En effet:

$1^{\circ}$ On a $|S|=2 n+2 n=4 n,|T|=4 n+1,|G|=8 n+2$, et la condition 5.6.1 est vérifiée.

$2^{\circ}$ On calcule $T+T$

$$
\begin{aligned}
T+T= & \{2, \ldots, 2 n ; 3 n+2, \ldots, 6 n+1 ; 7 n+3, \ldots, 9 n+1 ; \\
& 6 n+2, \ldots, 10 n+2 ; 10 n+3, \ldots, 13 n+2 ; \\
14 n+4, \ldots, 16 n+2\} & \\
= & \{2, \ldots, 2 n ; 3 n+2, \ldots, 6 n+1 ; 7 n+3, \ldots, 8 n+1 ; \\
& 0, \ldots, n-1 ; 6 n+2, \ldots, 8 n+1 ; 0, \ldots, 2 n ; \\
& 2 n+1, \ldots, 5 n ; 6 n+2, \ldots, 8 n\}=G .
\end{aligned}
$$

$3^{\circ}$ On calcule $S+T$

$$
\begin{aligned}
S+T= & \{n+2, \ldots, 4 n ; 4 n+2, \ldots, 8 n+1 ; 8 n+3, \ldots, 11 n+1 ; \\
& 5 n+3, \ldots, 8 n+1 ; 8 n+3, \ldots, 12 n+2 ; \\
12 n+4, \ldots, 15 n+2\} & \\
= & \{n+2, \ldots, 4 n ; 4 n+2, \ldots, 8 n+1 ; 1, \ldots, 3 n-1 ; \\
& 5 n+3, \ldots, 8 n+1 ; 1, \ldots, 4 n ; 4 n+2, \ldots, 7 n\} \\
= & \{1, \ldots, 4 n ; 4 n+2, \ldots, 8 n+1\} .
\end{aligned}
$$

Or $(4 n+1) \in T$ donc $(S+T) \cup T=G_{*}$.

De sorte que $(G ; S, T)$ est bien un groupe taillé.

$4^{\circ}$ On montre enfin que la condition 5.8.1 est vérifiée en établissant que $|A| \geq 2$ et $|B|=0$ (avec les notations du Lemme 5.8).

(1) On a $S=-S$. Donc, pour tout $x \in S$ on a $0 \in x+S$, d'où $0 \in A$. D'autre part, en posant $r=4 n+1$, on a $r+S=S$. Donc 
$A=\bigcap\{x+S ; x \in S\}=\bigcap\{x+r+S: x \in S\}=r+A$. Or 0 appartient à $A$ donc $r$ aussi et par suite on a $|A| \geq 2$.

(2) Pour montrer que l'ensemble $B=\bigcap\{x+T: x \in S \cup\{0\}\}$ est vide, on raisonne par l'absurde.

Si $a \in B$, on aurait, en particulier, $S \subset a-T=a+T$ et cela n'est pas possible: $S$ est composé de deux "arcs" chacun de "longueur" $2 n$; tandis que $T$ est composé de trois "arcs" de "longueurs" respectives $n, 2 n+1, n$.

C.Q.F.D.

On désigne par $K(n)$ le cogroupe associé.

On obtient ainsi une deuxième famille, $K(n), n \geq 1$, de cogroupes finis qui ne sont pas du type Utumi.

Le cogroupe $K(n)$ est d'ordre $2 m+1=16 n+5$. Le plus petit de la famille, $K(1)$, est d'ordre 21 .

5.10.3. Une troisième famille. On prend $n \geq 1, m=8 n+6$, $G=\mathbf{Z} / m \mathbf{Z}$,

$$
\begin{aligned}
& S=\{n+2, \ldots, 3 n+2 ; 5 n+4, \ldots, 7 n+4\}, \\
& T=\{1, \ldots, n+1 ; 3 n+3, \ldots, 5 n+3 ; 7 n+5, \ldots, 8 n+5\} .
\end{aligned}
$$

On obtient un groupe taillé $(G ; S, T)$ qui vérifie les conditions 5.6.1 et 5.7.1.

En effet:

$1^{\circ}$ On a $|S|=(2 n+1)+(2 n+1)=4 n+2,|T|=4 n+3$, $|G|=8 n+6$, et la condition 5.6.1 est vérifiée.

$2^{\circ}$ On calcule $T+T$ et on trouve $T+T=G$.

$3^{\circ}$ On calcule $S+T$ et on trouve $S+T=G_{*}$.

On voit ainsi que $(G ; S, T)$ est bien un groupe taillé.

$4^{\circ}$ La condition 5.7.1. L'ensemble $S$ est composé de deux "arcs"

$$
S_{1}=\{n+2, \ldots, 3 n+2\} \quad \text { et } \quad S_{2}=\{5 n+4, \ldots, 7 n+4\}
$$

tous deux de "longueur" $2 n+1$.

Supposons que $x+S=S$ et $x \neq 0$. On a alors $x+S_{1}=S_{2}$ et $x+S_{2}=S_{1}$ donc $2 x=0$ autrement dit $x=4 n+3$; mais pour cette valeur de $x$, on a $x+S_{1} \neq S_{2}$.

Ainsi, pour tout $x \neq 0$, on a $x+S \neq S$ donc $|S \cap(x+S)|<$ $|S|$.

C.Q.F.D.

On désigne par $L(n)$ le cogroupe associé. 
On a ainsi une troisième famille, $L(n), n \geq 1$, de cogroupes finis qui ne sont pas du type Utumi.

Le cogroupe $L(n)$ est d'ordre $2 m+1=16 n+13$. Le plus petit de la famille, $L(1)$, est d'ordre 29.

6. Comagma associé à un ensemble totalement ordonné. On se donne un ensemble $E$ totalement ordonné et pointé. On désigne par $\leq$ la relation d'ordre total et par 0 l'élément distingué de $E$. Pour tout élément $a$ de $E$, on pose

$$
\begin{aligned}
& S_{a}=\{x \in E \mid x<a\}, \\
& D_{a}=\{x \in E \mid x>a\},
\end{aligned}
$$

et l'on définit un hyperproduit sur $E$ comme suit:

$$
a b= \begin{cases}S_{2} & \text { si } b<0 \\ \{a\} & \text { si } b=0 \\ D_{2} & \text { si } b>0\end{cases}
$$

On obtient ainsi un hypermagma $H\langle E, 0\rangle$ associé à l'ensemble totalement ordonné pointé $\langle E, 0\rangle$.

De toute évidence 0 est unité scalaire à droite et, si $E=\{0\}$, l'hypermagma $H\langle E, 0\rangle$ est un groupe!

Lorsque $E \neq\{0\}$, on va donner des conditions nécessaires et suffisantes pour que $H\langle E, 0\rangle$ soit un cogroupe. Pour cela, on établit le lemme et le théorème suivants.

6.1. Lemme. On suppose $E \neq\{0\}$ et l'on pose $H=H\langle E, 0\rangle$. On a alors

$1^{\circ} x H=H$ pour tout $x$ de $H$ ssi 0 n'est ni premier ni dernier élément.

$2^{\circ} H y=H$ pour tout $y$ de $H$ ssi $E$ n'a ni premier ni dernier élément.

La démonstration de la première équivalence est triviale. Celle de la seconde repose sur les égalités suivantes:

$$
H y= \begin{cases}H & \text { si } y=0 \\ \bigcup_{x \in H} S_{x} & \text { si } y<0 \\ \bigcup_{x \in H} D_{x} & \text { si } y>0\end{cases}
$$

Si $p$ est le premier élément de $E$, distinguons deux cas:

1. S'il existe $y>0$, alors $H y=\bigcup_{x \in H} D_{x}$ et il s'ensuit $p \notin H y$. 
2. S'il n'existe pas d'élément $y>0$, alors on a $p<0$ et $0 \notin S_{0}=$ $\mathrm{Hp}$.

Ainsi, la reproductibilité à droite de $H$ nécessite que $E$ n'ait pas de premier élément et, de même, de dernier élément. Réciproquement, si $E$ n'a ni premier ni dernier élément il s'ensuit

$$
\begin{array}{ll}
H y & =\bigcup_{x \in H} S_{x}=H \quad \text { pour tout } y<0, \\
H y & =\bigcup_{x \in H} D_{x}=H \quad \text { pour tout } y>0, \\
H 0 & =H .
\end{array}
$$

6.2. Théorème. On suppose $E \neq\{0\}$. Soit $H=H\langle E, 0\rangle$. Les trois assertions suivantes sont alors équivalentes:

(i) L'hypermagma $H$ est un hypergroupe.

(ii) L'ensemble totalement ordonné $E$ est dense et n'a ni premier ni dernier élément.

(iii) L'hypermagma $H$ est un hypergroupe de type $C$.

Démonstration. L'assertion (iii) implique trivalement (i).

Réciproquement, si $H$ est un hypergroupe, pour tous $a, b$ et $c$ de $H$, l'ensemble $a b \cap a c$ est non vide seulement dans les trois cas suivants:

$$
\begin{array}{ll}
b=c=0 & \text { alors } a b=\{a\}=a c, \\
b>0 \text { et } c>0 & \text { alors } a b=D_{a}=a c, \\
b<0 \text { et } c<0 & \text { alors } a b=S_{a}=a c .
\end{array}
$$

Ce qui montre que $H$ est de type $C$, et donc (i) implique (iii). On établit maintenant l'équivalence de (i) et (ii).

Si $H$ est un hypergroupe, par le Lemme 6.1, l'ensemble $E$ n'a ni premier ni dernier élément et si $E$ est non dense il existe au moins un élément $a$ de $E$ ayant un successeur immédiat $c=a^{+}$et alors, pour tout élément $b>0$ de $E$ (dont l'existence est assurée par l'absence d'un dernier élément) on a:

$$
a(b b)=a D_{b}=D_{a} \neq D_{c}=\bigcup_{x>a} D_{x}=(a b) b
$$

ce qui est absurde!

Inversement, si $E(\neq\{0\})$ est dense sans premier ni dernier élément, le Lemme 6.1 assure la reproductibilité de l'hyperloi de $H$. 
Vérifions-en l'associativité. Pour cela, $a, b$ et $c$ étant trois éléments de $H$, on pose $u=a(b c)$ et $v=(a b) c$.

Pour $c=0$ ou $b=0$ on vérifie aisément $u=v$. Pour $c \neq 0$ et $b \neq 0$ distinguons 4 cas:

$b>0$ et $c>0$ : On a $b c=D_{b} \neq \varnothing$, d'où:

$$
\begin{aligned}
& u=a D_{b}=D_{a} \\
& v=D_{a} c=\bigcup_{x>a} D_{x} .
\end{aligned}
$$

L'inclusion $v \subset u$ est immédiate, et, si $\alpha$ est dans $u$, par la densité de $E$, il existe $\beta$ vérifiant $a<\beta<\alpha$ d'où l'on tire $\alpha \in D_{\beta} \subset v$ et l'on conclut $u=v$. Ainsi pour $b>0$ et $c>0$, il vient $a(b c)=D_{a}=$ $(a b) c$. On traite de même les trois cas restants:

$$
\begin{array}{ll}
b>0 \text { et } c<0, & a(b c)=H=(a b) c \\
b<0 \text { et } c>0, & a(b c)=H=(a b) c \\
b<0 \text { et } c<0, & a(b c)=S_{a}=(a b) c
\end{array}
$$

On en arrive maintenant aux conditions nécessaires et suffisantes annoncées en début de paragraphe.

6.3. ThÉORÈME. Si $E \neq\{0\}$, alors $H=H\langle E, 0\rangle$ est un cogroupe ssi:

1. L'ensemble $E$ est dense, sans premier ni dernier élément et

2. on a $\left|S_{x}\right|=\left|S_{0}\right|$ et $\left|D_{x}\right|=\left|D_{0}\right|$ pour tout $x$ de $E$.

En effet, la condition 1 est nécessaire et suffisante pour que $H$ soit un hypergroupe de type $C$ (c'est le théorème précédent) et, pour tout $x$ et $y$ de $H$, on a

$$
|x y|= \begin{cases}\left|S_{x}\right| & \text { si } y<0 \\ 1 & \text { si } y=0 \\ \left|D_{x}\right| & \text { si } y>0\end{cases}
$$

Ainsi, $|x y|$ est indépendant de $x$ si et seulement si tout $x$ de $H$ satisfait les égalités

$$
\left|S_{x}\right|=\left|S_{0}\right| \quad \text { et } \quad\left|D_{x}\right|=\left|D_{0}\right| \text {. }
$$

La seconde partie de ce paragraphe est consacrée à la détermination des multiplicateurs de l'hypermagma $H\langle E, 0\rangle$. 
6.4. ThÉORÈm. Pour qu'une application $\theta: E \rightarrow E$ soit un multiplicateur de $H\langle E, 0\rangle$, il faut et il suffit que $\theta$ soit un automorphisme d'ordre de $E$.

Supposons que $\theta$ soit un multiplicateur de $H\langle E, 0\rangle$. C'est une permutation de $E$. On va établir que $\theta$ est un automorphisme d'ordre de $E$, c'est-à-dire:

$$
x<y \Rightarrow \theta(x)<\theta(y) .
$$

On distingue trois cas:

$x \geq 0$. Alors on a $y>0$ et $y \in x y=D_{x}$ mais d'autre part

$$
\theta(x y)=\theta(x) y=D_{\theta(x)}
$$

donc $\theta(y)$ appartient à $D_{\theta(x)}$ soit $\theta(y)>\theta(x)$.

$y \leq 0$. Comme ci-dessus: $x<0$ et $x \in y x=S_{y}$ mais $\theta(y x)=$ $\theta(y) x=S_{\theta(y)}$, donc $\theta(x) \in S_{\theta(y)}$ soit $\theta(x)<\theta(y)$.

$x<0$ et $y>0$. Alors on a $y \in x y=D_{x}$ d'où $\theta(y) \in \theta(x y)=$ $\theta(x) y=D_{\theta(x)}$. Ainsi $\theta(y)>\theta(x)$.

Réciproquement, supposons que $\theta$ soit un automorphisme d'ordre de $E$ et considérons deux éléments $x$ et $y$ de $E$. On va montrer l'égalité $\theta(x y)=\theta(x) y$.

Pour cela, on distingue encore trois cas:

$y=0$. Alors on a $x y=x$, d'où $\theta(x y)=\theta(x)$ donc $\theta(x y)=$ $\theta(x) y$.

$y>0$. Il s'ensuit $x y=D_{x}$ et $\theta(x) y=D_{\theta(x)}$

$$
\text { d'où } \begin{aligned}
\theta(x y) & =\theta\left(D_{x}\right)=\theta(\{z: z>x\}) \\
& =\{\theta(z): \theta(z)>\theta(x)\} \\
& =D_{\theta(x)}=\theta(x) y .
\end{aligned}
$$

$y<0$. Comme ci-dessus on obtient

$$
\theta(x y)=\theta\left(S_{x}\right)=S_{\theta(x)}=\theta(x) y . \quad \text { C.Q.F.D. }
$$

6.5. Corollaire. Si l'hypermagma $H\langle E, 0\rangle$ est un cogroupe alors les deux énoncés suivants sont équivalents:

(i) Le cogroupe $H\langle E, 0\rangle$ est du type Utumi.

(ii) Pour chaque élément $x$ de $E$, il existe au moins un automorphisme d'ordre $f$ de $E$ vérifiant $f(0)=x$.

Le corollaire est immédiat compte tenu des résultats 4.7 et 6.4 . 
6.6. Une famille de cogroupes. On présente maintenant une famille de cogroupes $C(\lambda)$ associés des ensembles totalement ordonnés $E(\lambda)$ et qui servent d'exemples et de contre-exemples.

Pour chaque ordinal $\lambda>0$, on considère la somme ordinale de $\lambda$ copies de $\mathbf{R}$

$$
E(\lambda)=\sum_{i<\lambda} \mathbf{R}_{i}
$$

$C$ 'est un ensemble totalement ordonné dense, sans premier ni dernier élément. On pointe l'ensemble $E(\lambda)$ en $0=(o, o)$, l'origine dans la première copie $\mathbf{R}_{o}$ de $\mathbf{R}$.

On considère alors l'hypermagma associé $C(\lambda)=H\langle E(\lambda), 0\rangle$. C'est un hypergroupe de type $C$, en vertu du Théorème 6.2.

Commençons par observer ceci.

1. Tout automorphisme $f$ de l'ensemble totalement ordonné $E(\lambda)$ transforme chaque copie $\mathbf{R}_{i}$ en elle-même.

En effet: On remarque tout d'abord que $f\left(\mathbf{R}_{i}\right)$ qui est isomorphe à $\mathbf{R}$ ne saurait couper deux composantes distinctes de $E(\lambda)$ car, sinon, il couperait deux composantes consécutives $\mathbf{R}_{j}$ et $\mathbf{R}_{j+1}$ ce qui déterminerait une coupure sur $f\left(\mathbf{R}_{i}\right)$ !

Ainsi, pour chaque $i<\lambda$, il existe $\varphi(i)<\lambda$ tel que $f\left(\mathbf{R}_{i}\right) \subset \mathbf{R}_{\varphi(i)}$. Et puisque l'on a de même $f^{-1}\left(\mathbf{R}_{\varphi(i)}\right) \subset \mathbf{R}_{i}$, on conclut que $f\left(\mathbf{R}_{i}\right)=$ $\mathbf{R}_{\varphi(i)}$ et $\varphi$ est une application surjective strictement croissante de l'ordinal $\lambda$ sur lui-même. On a donc $\varphi(i)=i$.

C.Q.F.D.

On désigne par $\gamma$ le cardinal du continu, $\gamma=2^{\aleph_{0}}$, et on observe ensuite ceci.

2. Pour $0<|\lambda| \leq \gamma$, l'hypergroupe $C(\lambda)$ est un cogroupe.

En effet: Il suffit de montrer que, pour chaque $x \in E(\lambda)$, on a

$$
\left|S_{x}\right|=\left|D_{x}\right|=\gamma
$$

et d'appliquer le Théorème 6.3.

Or, si $x$ appartient à la copie $\mathbf{R}_{i}(i<\lambda)$, on a d'une part

d'autre part

$$
\gamma \leq\left|S_{x}\right| \leq\left|\sum_{j \leq i} \mathbf{R}_{j}\right| \leq \gamma \gamma=\gamma
$$

D’où le résultat.

$$
\gamma \geq|\lambda| \gamma \geq\left|D_{x}\right| \geq\left|\sum_{i<j<\lambda} \mathbf{R}_{j}\right| \geq \gamma .
$$


3. Ainsi, pour $2 \leq|\lambda| \leq \gamma$, l'hypergroupe $C(\lambda)$ est un cogroupe qui n'est pas du type Utumi. C'est une conséquence de ce qui précède et du Corollaire 6.5.

On pourra observer, enfin, que $C(1)$ est un $D$-hypergroupe et que $C(2)$ est un exemple simple et commode de cogroupe qui n'est pas du type Utumi.

6.7. Un dernier exemple. L'un des hypergroupes de la famille $C(\lambda)$ définie ci-dessus présente une particularité sur laquelle nous dirons deux mots pour finir. Il s'agit de $C(\theta)$ où $\theta=\gamma^{+}$est le cardinal successeur immédiat du cardinal du continu $\gamma=2^{\aleph_{0}}$.

1. L'hypergroupe $C(\theta)$ est un cogroupe. Plus précisément, pour chaque $x \in E(\theta)$, on a

$$
\left|S_{x}\right|=\gamma \quad \text { et } \quad\left|D_{x}\right|=\theta
$$

En effet: Si $x$ appartient à la copie $\mathbf{R}_{i}(i<\theta)$, on a

$$
\gamma \leq\left|S_{x}\right| \leq\left|\sum_{j \leq i} \mathbf{R}_{j}\right| \leq \gamma \gamma=\gamma
$$

car l'ensemble $\{j \mid j \leq i\}$ a pour cardinal $\gamma$ au plus. D'où $\left|S_{x}\right|=\gamma$.

D'autre part,

$$
D_{x} \supset \sum_{i<j<\theta} \mathbf{R}_{j}
$$

et l'ensemble $\{j \mid i<j<\theta\}$ a pour cardinal $\theta$ donc

$$
\theta \geq\left|D_{x}\right| \geq \theta \cdot \gamma=\theta
$$

D'où

$$
\left|D_{x}\right|=\theta \text {. }
$$

Le Théorème 6.3 permet de conclure que $C(\theta)$ est bien un cogroupe.

2. Le cogroupe $C(\theta)$ n'est pas du type Utumi.

C.Q.F.D.

C'est une conséquence du Corollaire 6.5 et de 6.6.1.

3. Pour chaque couple $(x, y)$ d'éléments non nuls de $C(\theta)$ tels que $0 \in x y$, les deux ensembles $0 x$ et $0 y$ n'ont pas le même cardinal. 
En effet: Les deux éléments $x$ et $y$ ne sont pas du même côté par rapport à 0 . Supposons donc que l'on ait

$$
x<0 \text { et } y>0 \text {. }
$$

Alors

$$
0 x=S_{0} \quad \text { et } \quad 0 y=D_{0}
$$

de sorte que l'on a

$$
|0 x|=\gamma \neq \theta=|0 y| .
$$

De même, si l'on avait

$$
x>0 \text { et } y<0
$$

on aurait

$$
|0 x|=\theta \neq \gamma=|0 y| \text {. }
$$

Cette dernière particularité ne se recontre jamais dans les cogroupes finis. On relira à ce sujet le Théorème 5 de Eaton [2]. On notera, au passage, que la démonstration donnée par Eaton, quelque peu cursive, ne saurait s'appliquer aux cogroupes infinis.

\section{Appendices}

Démonstration des conditions d'associativité 5.4.

On se donne des éléments $a, b, c$ de $H$.

On pose $u=a(b c), v=(a b) c$.

1. Pour $c=e$ : on a $u=a(b e)=a b$ et $v=(a b) e=a b$. Donc $u=v$.

2. Pour $b=e, c \neq e:$ On a

$$
u=a(e c)=\left\{\begin{array}{ll}
a G & \text { si } c \in G \\
a \bar{G} & \text { si } c \in \bar{G}
\end{array}\right\}=a c
$$

$v=(a e) c=a c$. Donc $u=v$ à nouveau.

3. Pour $a=e, b \neq e, c \neq e$ : On a $u=e(b c)$ et $v=(e b) c$. D'où le tableau suivant qui donne aussi bien la valeur de $u$ que la valeur de $v$. On constate donc à nouveau l'égalité $u=v$.

\begin{tabular}{|c|c|c|}
\hline$b$ & $G$ & $\bar{G}$ \\
\hline$G$ & $H$ & $H \backslash\{e\}$ \\
\hline $\bar{G}$ & $H \backslash\{e\}$ & $H$ \\
\hline
\end{tabular}


En résumé, on a $u=v$ si l'un quelconque des éléments $a, b, c$ est égal à $e$. Il s'ensuit donc que, pour toutes parties $A$ et $B$ de $H$, on a $A B=(A e) B=A(e B)$, ce qui permet d'écrire simplement $A B=A e B$.

4. Pour $a \neq e, b \neq e, c \neq e$ : il y a $2 \times 2 \times 2=8$ cas à examiner que nous regroupons en $4+4$ suivant que $a$ est dans $G$ ou dans $\bar{G}$.

4.1. Cas où $a \in G$. Il se décompose en quatre cas.

4.1.1. Cas $b \in G, c \in G$. En vertu de 3 on a $u=a(b c)=a e(b c)=$ $a H=H$ et

$$
\begin{aligned}
v= & (a b) c=(\{e\} \cup(a+C) \cup \overline{(a+D)}) G \\
= & G \cup\{e\} \cup(a+C+C) \cup \overline{(a+C+D)} \\
& \cup\left(a+D+E^{\prime}\right) \cup \overline{\left(a+D+F^{\prime}\right)} .
\end{aligned}
$$

Ainsi, on aura $u=v$ si et seulement si l'on a la condition

$P(a)$

$$
(a+C+D) \cup\left(a+D+F^{\prime}\right)=G .
$$

Pour $a=0$, cette condition s'écrit

$$
(C+D) \cup\left(D+F^{\prime}\right)=G .
$$

Et l'on voit bien que la condition $P(a)$ est satisfaite pour tout $a \in G$ si et seulement si la condition (1) est satisfaite.

On procède de même pour les trois autres cas.

4.1.2. Cas $b \in G, c \in \bar{G} . \quad u=a(b c)=a e(b c)=a(H \backslash\{e\})=$ $H \backslash\{a\} . \mathrm{Et}$

$$
\begin{aligned}
v= & (a b) c=(\{e\} \cup(a+C) \cup \overline{(a+D)}) \bar{G} \\
= & \bar{G} \cup\left(a+C+C^{\prime}\right) \cup \overline{\left(a+C+D^{\prime}\right)} \cup\{e\} \\
& \cup(a+D+E) \cup \overline{(a+D+F) .}
\end{aligned}
$$

On aura donc $u=v$ pour tout $a \in G$, si et seulement si l'on a

$$
\left(C+C^{\prime}\right) \cup(D+E)=G_{*} \text {. }
$$

4.1.3. Cas $b \in \bar{G}, c \in G . u=a(b c)=H \backslash\{a\}$. Et

$$
\begin{aligned}
v= & (a b) c=\left(\left(a+C^{\prime}\right) \cup \overline{\left(a+D^{\prime}\right)}\right) G \\
= & \{e\} \cup\left(a+C^{\prime}+C\right) \cup \overline{\left(a+C^{\prime}+D\right)} \\
& \cup\left(a+D^{\prime}+E^{\prime}\right) \cup \overline{\left(a+D^{\prime}+F^{\prime}\right)} .
\end{aligned}
$$


On aura donc $u=v$ pour tout $a \in G$ si et seulement si les deux conditions suivantes sont réunies

$$
\begin{gathered}
\left(C^{\prime}+C\right) \cup\left(D^{\prime}+E^{\prime}\right)=G_{*}, \\
\left(C^{\prime}+D\right) \cup\left(D^{\prime}+F^{\prime}\right)=G .
\end{gathered}
$$

4.1.4. Cas $b \in \bar{G}, c \in \bar{G} . u=a(b c)=H$. Et

$$
\begin{aligned}
v= & (a b) c=\left(\left(a+C^{\prime}\right) \cup \overline{\left(a+D^{\prime}\right)}\right) \bar{G} \\
= & \left(a+C^{\prime}+C^{\prime}\right) \cup \overline{\left(a+C^{\prime}+D^{\prime}\right)} \cup\{e\} \\
& \cup\left(a+D^{\prime}+E\right) \cup \overline{\left(a+D^{\prime}+F\right)} .
\end{aligned}
$$

Ainsi $u=v$ pour tout $a \in G$, si et seulement si les deux conditions suivantes sont remplies

$$
\begin{aligned}
& \left(C^{\prime}+C^{\prime}\right) \cup\left(D^{\prime}+E\right)=G, \\
& \left(C^{\prime}+D^{\prime}\right) \cup\left(D^{\prime}+F\right)=G .
\end{aligned}
$$

4.2. Cas où $a \in \bar{G}$. Il s'obtient par "symétrie" en échangeant d'une part $C$ et $F$, d'autre part $D$ et $E$.

C.Q.F.D.

TABLE DU COGROUPE $H(2)$ (paragraphe 5.10.1)

\begin{tabular}{|l|llllllll|llllllll|}
\hline$e$ & 0 & 1 & 2 & 3 & 4 & 5 & 6 & 7 & $\overline{0}$ & $\overline{1}$ & $\overline{2}$ & $\overline{3}$ & $\overline{4}$ & $\overline{5}$ & $\overline{6}$ & $\overline{7}$ \\
\hline 0 & $e$ & 3 & 4 & 5 & $\overline{0}$ & $\overline{3}$ & $\overline{4}$ & $\overline{5}$ & 1 & 2 & 6 & 7 & $\overline{1}$ & $\overline{2}$ & $\overline{6}$ & $\overline{7}$ \\
\hline 1 & $e$ & 4 & 5 & 6 & $\overline{1}$ & $\overline{4}$ & $\overline{5}$ & $\overline{6}$ & 2 & 3 & 7 & 0 & $\overline{2}$ & $\overline{3}$ & $\overline{7}$ & $\overline{0}$ \\
\hline 2 & $e$ & 5 & 6 & 7 & $\overline{2}$ & $\overline{5}$ & $\overline{6}$ & $\overline{7}$ & 3 & 4 & 0 & 1 & $\overline{3}$ & $\overline{4}$ & $\overline{0}$ & $\overline{1}$ \\
\hline 3 & $e$ & 6 & 7 & 0 & $\overline{3}$ & $\overline{6}$ & $\overline{7}$ & $\overline{0}$ & 4 & 5 & 1 & 2 & $\overline{4}$ & $\overline{5}$ & $\overline{1}$ & $\overline{2}$ \\
\hline 4 & $e$ & 7 & 0 & 1 & $\overline{4}$ & $\overline{7}$ & $\overline{0}$ & $\overline{1}$ & 5 & 6 & 2 & 3 & $\overline{5}$ & $\overline{6}$ & $\overline{2}$ & $\overline{3}$ \\
\hline 5 & $e$ & 0 & 1 & 2 & $\overline{5}$ & $\overline{0}$ & $\overline{1}$ & $\overline{2}$ & 6 & 7 & 3 & 4 & $\overline{6}$ & $\overline{7}$ & $\overline{3}$ & $\overline{4}$ \\
\hline 6 & $e$ & 1 & 2 & 3 & $\overline{6}$ & $\overline{1}$ & $\overline{2}$ & $\overline{3}$ & 7 & 0 & 4 & 5 & $\overline{7}$ & $\overline{0}$ & $\overline{4}$ & $\overline{5}$ \\
\hline 7 & $e$ & 2 & 3 & 4 & $\overline{7}$ & $\overline{2}$ & $\overline{3}$ & $\overline{4}$ & 0 & 1 & 5 & 6 & $\overline{0}$ & $\overline{1}$ & $\overline{5}$ & $\overline{6}$ \\
\hline$\overline{0}$ & 0 & 3 & 4 & 5 & $\overline{1}$ & $\overline{2}$ & $\overline{6}$ & $\overline{7}$ & $e$ & 1 & 2 & 6 & 7 & $\overline{3}$ & $\overline{4}$ & $\overline{5}$ \\
\hline$\overline{1}$ & 1 & 4 & 5 & 6 & $\overline{2}$ & $\overline{3}$ & $\overline{7}$ & $\overline{0}$ & $e$ & 2 & 3 & 7 & 0 & $\overline{4}$ & $\overline{5}$ & $\overline{6}$ \\
\hline$\overline{2}$ & 2 & 5 & 6 & 7 & $\overline{3}$ & $\overline{4}$ & $\overline{0}$ & $\overline{1}$ & $e$ & 3 & 4 & 0 & 1 & $\overline{5}$ & $\overline{6}$ & $\overline{7}$ \\
\hline$\overline{3}$ & 3 & 6 & 7 & 0 & $\overline{4}$ & $\overline{5}$ & $\overline{1}$ & $\overline{2}$ & $e$ & 4 & 5 & 1 & 2 & $\overline{6}$ & $\overline{7}$ & $\overline{0}$ \\
\hline$\overline{4}$ & 4 & 7 & 0 & 1 & $\overline{5}$ & $\overline{6}$ & $\overline{2}$ & $\overline{3}$ & $e$ & 5 & 6 & 2 & 3 & $\overline{7}$ & $\overline{0}$ & $\overline{1}$ \\
\hline$\overline{5}$ & 5 & 0 & 1 & 2 & $\overline{6}$ & $\overline{7}$ & $\overline{3}$ & $\overline{4}$ & $e$ & 6 & 7 & 3 & 4 & $\overline{0}$ & $\overline{1}$ & $\overline{2}$ \\
\hline$\overline{6}$ & 6 & 1 & 2 & 3 & $\overline{7}$ & $\overline{0}$ & $\overline{4}$ & $\overline{5}$ & $e$ & 7 & 0 & 4 & 5 & $\overline{1}$ & $\overline{2}$ & $\overline{3}$ \\
\hline$\overline{7}$ & 7 & 2 & 3 & 4 & $\overline{0}$ & $\overline{1}$ & $\overline{5}$ & $\overline{6}$ & $e$ & 0 & 1 & 5 & 6 & $\overline{2}$ & $\overline{3}$ & $\overline{4}$ \\
\hline
\end{tabular}




$$
\begin{array}{ccc}
G=Z / 8 Z & S=\{3,4,5\} & T=\{1,2,6,7\} \\
C=F=S & D=S \cup\{0\} & E=T
\end{array}
$$

\section{BIBLIOGRAPHIE}

[1] S. D. Comer, Polygroups derived from cogroups, J. Algebra, 89 (1984), $\mathrm{n}^{\circ} 2$, 397-405.

[2] J. E. Eaton, Theory of cogroups, Duke Math. J., 6 (1940), 101-107.

[3] L. Haddad et Y. Sureau, Les cogroupes et les D-hypergroupes, J. Algebra, 118 $\mathrm{n}^{\circ} 2,(1988), 468-476$.

[4] M. Krasner, Sur la théorie de la ramification des idéaux des corps non-galoisiens de nombres algébriques (Thèse, Paris), Mémoires (in $4^{\circ}$ ) de l'Académie de Belgique (Classe des Sciences), 11 (1937), $\mathrm{n}^{\circ} 4,1-110$.

[5] _ La caractérisation des hypergroupes de classes et le problème de Schreier dans ces hypergroupes, C. R. Acad. Sci., Paris, 212 (1941), 948-950.

[6] __ Rectification à ma Note précédente et quelques nouvelles contributions à la théorie des hypergroupes, C. R. Acad. Sci., Paris, 218 (1944), 542-544.

[7] Y. Sureau, Contribution à la Théorie des Hypergroupes et Hypergroupes Opérant Transitivement sur un Ensemble, Thèse de Doctorat d'Etat, Univ. de Clermont II, 1980.

[8] _-, Hypergroupes de type C, Conférence Taormina, 1983.

[9] Y. Utumi, On hypergroups of group right cosets, Osaka Math. J., 1 n ${ }^{\circ} 1$, (1949), 73-80.

Received October 5, 1988.

Université Blaise Pascal

F-63177 Aubiekre Cedex, France 


\section{PACIFIC JOURNAL OF MATHEMATICS EDITORS}

\author{
V. S. VARAdarajaN \\ (Managing Editor) \\ University of California \\ Los Angeles, CA 90024-1555-05 \\ Herbert Clemens \\ University of Utah \\ Salt Lake City, UT 84112 \\ Thomas ENRIGHT \\ University of California, San Diego \\ La Jolla, CA 92093
}

R. FINN

Stanford University

Stanford, CA 94305

HermanN FlaschKa

University of Arizona

Tucson, AZ 85721

VAUGHan F. R. Jones

University of California

Berkeley, CA 94720

STEVEN KeRCKHOFF

Stanford University

Stanford, CA 94305
C. C. Moore University of California Berkeley, CA 94720

MARTIN SCHARLEMANN University of California Santa Barbara, CA 93106

\section{HAROLD STARK}

University of California, San Diego La Jolla, CA 92093

\section{ASSOCIATE EDITORS}
R. ARENS
E. F. BECKeNBACH
B. H. NeumanN
F. WolF
(1904-1989)
K. Yoshida (1906-1982)

\section{SUPPORTING INSTITUTIONS}

UNIVERSITY OF ARIZONA

UNIVERSITY OF BRITISH COLUMBIA

CALIFORNIA INSTITUTE OF TECHNOLOGY

UNIVERSITY OF CALIFORNIA

MONTANA STATE UNIVERSITY

UNIVERSITY OF NEVADA, RENO

NEW MEXICO STATE UNIVERSITY

OREGON STATE UNIVERSITY
UNIVERSITY OF OREGON

UNIVERSITY OF SOUTHERN CALIFORNIA

STANFORD UNIVERSITY

UNIVERSITY OF HAWAII

UNIVERSITY OF TOKYO

UNIVERSITY OF UTAH

WASHINGTON STATE UNIVERSITY

UNIVERSITY OF WASHINGTON 


\section{Pacific Journal of Mathematics}

Vol. 145, No. 1 September, 1990

Sheldon Jay Axler and Allen Lowell Shields, Extensions of harmonic and

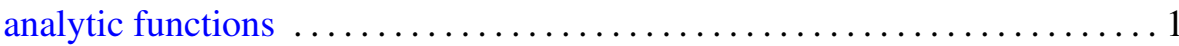

Labib Haddad and Yves Sureau, Les cogroupes et la construction de

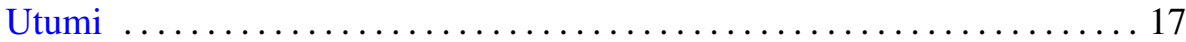

John Hutchinson, Poincaré-Sobolev and related inequalities for submanifolds of $\mathbf{R}^{N}$

Yuk Jaum Leung and Glenn E. Schober, Some coefficient problems and

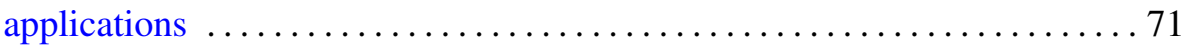

Daniel Ruberman, Seifert surfaces of knots in $S^{4} \ldots \ldots \ldots \ldots \ldots \ldots \ldots \ldots$

Joel Harold Shapiro and Carl Sundberg, Isolation amongst the

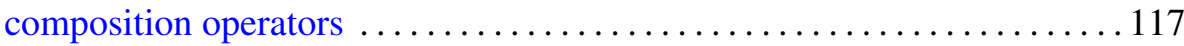

Hans Wenzl, Representations of braid groups and the quantum Yang-Baxter

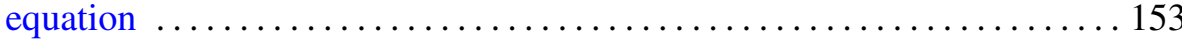

Shuang Zhang, Diagonalizing projections in multiplier algebras and in matrices over a $C^{*}$-algebra 